\title{
The Unexpected Rapid Intensification of Tropical Cyclones in Moderate Vertical Wind Shear. Part I: Overview and Observations ${ }^{\mathscr{O}}$
}

\author{
David R. Ryglicki, Joshua H. Cossuth, Daniel Hodyss, and James D. Doyle \\ Naval Research Laboratory, Monterey, California
}

(Manuscript received 19 January 2018, in final form 7 August 2018)

\begin{abstract}
A satellite-based investigation is performed of a class of tropical cyclones (TCs) that unexpectedly undergo rapid intensification (RI) in moderate vertical wind shear between 5 and $10 \mathrm{~m} \mathrm{~s}^{-1}$ calculated as $200-850-\mathrm{hPa}$ shear. This study makes use of both infrared $(\mathrm{IR} ; 11 \mu \mathrm{m})$ and water vapor $(\mathrm{WV} ; 6.5 \mu \mathrm{m})$ geostationary satellite data, the Statistical Hurricane Prediction Intensity System (SHIPS), and model reanalyses to highlight commonalities of the six TCs. The commonalities serve as predictive guides for forecasters and common features that can be used to constrain and verify idealized modeling studies. Each of the TCs exhibits a convective cloud structure that is identified as a tilt-modulated convective asymmetry (TCA). These TCAs share similar shapes, upshear-relative positions, and IR cloud-top temperatures (below $-70^{\circ} \mathrm{C}$ ). They pulse over the core of the TC with a periodicity of between 4 and $8 \mathrm{~h}$. Using WV satellite imagery, two additional features identified are asymmetric warming/drying upshear of the TC relative to downshear, as well as radially thin arc-shaped clouds on the upshear side. The WV brightness temperatures of these arcs are between $-40^{\circ}$ and $-60^{\circ} \mathrm{C}$. All of the TCs are sheared by upper-level anticyclones, which limits the strongest environmental winds to near the tropopause.
\end{abstract}

\section{Introduction}

\section{a. Background}

Tropical cyclones (TCs) that undergo rapid intensification [RI; an increase of maximum sustained surface winds of at least $30 \mathrm{kt}\left(15 \mathrm{~m} \mathrm{~s}^{-1} ; 1 \mathrm{kt} \approx 0.5144 \mathrm{~m} \mathrm{~s}^{-1}\right)$ in a $24-\mathrm{h}$ period] are difficult to predict (Krishnamurti et al. 2005). Numerous studies have identified the larger-scale factors that are positively correlated with RI: factors such as warm sea surface temperatures (SSTs), weak vertical wind shear, and large upper-level divergence (e.g., Gray 1968; Kaplan et al. 2010). While not necessarily causal, these large-scale factors at least provide clues for how TCs evolve with respect to their environments. Despite this level of insight, there remain outlier TCs that undergo RI in the presence of what are otherwise perceived as unfavorable conditions for RI, so we deem it necessary to investigate TCs that exist outside the expected norms of RI.

Supplemental information related to this paper is available at the Journals Online website: https://doi.org/10.1175/MWR-D-18-0020.s1.

Corresponding author: David R. Ryglicki, david.ryglicki@nrlmry. navy.mil
Recently, the "unfavorable condition" that is most often associated with intensity change is vertical wind shear (Tao and Zhang 2015); therefore, the focus of this study is to characterize a select group of TCs that undergo RI in moderate 200-850-hPa vertical wind shear [e.g., 10-22 kt $\left(5-11 \mathrm{~m} \mathrm{~s}^{-1}\right)$; Reasor et al. 2009; Rios-Berrios and Torn 2017]. This set of TCs, which develop outside the expected climatological norms of RI, all share similar features in satellite and reanalysis datasets (i.e., episodic pulsing, persistent far-field warm-cold asymmetry as measured via satellite, consistent upper-level forcing mechanism), and this study identifies and quantifies a set of key common features for this class of TCs. The analyses in this study primarily rely on satellite observations, since these data typically provide the most complete spatial and temporal coverage over the storms, given that aircraft observations of TCs are rare in the eastern North Pacific (EPAC) and western Pacific (WPAC; Knabb et al. 2008). Without in situ reconnaissance data, intensity estimations and analyses are primarily reliant upon the interpretation of satellite measurements, with the majority of those measurements coming from geostationary satellites.

Geostationary satellite measurements have been used for a host of TC applications. Perhaps the most well-known 
application of geostationary satellite imagery interpretation, at least in operational realms, is the Dvorak technique (Dvorak 1975, 1984). Dvorak developed a large-scale climatological view of TCs, aggregating successional satellite images over multiple years to construct guidelines for forecasters by relating specific features with current intensity. It has since been refined several times to add objective methods for estimating TC strength (Dvorak 1984; Zehr 1989; Guard et al. 1992; Velden et al. 1998; Olander et al. 2004; Olander and Velden 2007). A modern version of the Dvorak technique, the advanced Dvorak technique (ADT), has even been used as a reanalysis tool for historical TC studies (Velden et al. 2017). In addition to the Dvorak technique, geostationary satellite observations have been used in other ways to document TC evolution and structure. Black and Anthes (1971) and Merrill and Velden (1996) used satellite-track winds to quantify the structure of the upper-level outflow of a TC. These satellite-track winds are now more commonly known as atmospheric motion vectors (AMVs; Velden et al. 1997), and they are widely used for a variety of qualitative and quantitative analyses, such as the aforementioned studies of TC outflow or computing AMV-based vertical wind shear values (e.g., Velden and Sears 2014). In addition to AMVs, geostationary satellite measurements have also been used in a diagnostic way to physically characterize certain phenomena of TCs. Olander and Velden (2009) used the difference between infrared and water vapor bands to investigate overshooting tops in convection, while Griffin et al. (2016) used infrared (IR) imagery augmented with CloudSat and MODIS imagery to further investigate overshooting tops. Kossin (2002) and Dunion et al. (2014) have examined both the structure and the temporal evolution of the diurnal cycle of mature TCs using geostationary satellite imagery. Lander (1994) used satellite imagery to document the interplay between a large monsoon gyre in the western Pacific and tropical cyclogenesis. The aforementioned works are only a few examples illustrating how satellite observations and interpretations continue to be important for TC analyses.

One of the limiting factors on TC intensification first identified by Gray (1968) was the influence of vertical wind shear and its ability to distort the cloud field (Dvorak 1975), thus indicating an arrested development of the TC. The inhibiting characteristic of vertical wind shear on the growth and sustenance of a TC has made it a key component of statistical TC intensity models, such as the Statistical Hurricane Intensity Prediction System (SHIPS; DeMaria et al. 2005) and its RI offshoot (SHIPS-RII; Kaplan et al. 2010). Given small 200-850-hPa vertical wind shear values, large upper-level divergence values, high low-level relative humidity values, and large intensification potential, SHIPS-RII will most likely forecast that a TC will undergo RI. We will henceforth call this scenario "classic" RI, since it reflects a traditional way of considering a favorable growth environment. TCs defying this classical scenario primarily with regards to shear, thus violating expectations of both SHIPS-RII and the Dvorak technique, have also been observed, and this work will focus on this "atypical" class of TCs. Six cases have been observed that all share similar characteristics in both IR and water vapor (WV) satellite observations, SHIPS-RII parameters, and upper-level synoptic situations, but were all poorly forecast by both official forecasters and operational models. They are 1997 EPAC Guillermo, 2008 EPAC Hernan, 2008 EPAC Norbert, 2012 EPAC Fabio, 2015 EPAC Hilda, and 2015 North Atlantic (NATL) Joaquin. These choices are limited by climatological studies using the SHIPS database, so we have not performed any inspections of other basins as of yet.

The most prominent feature in the satellite imagery of this atypical class, to be described further in this manuscript, is a period of time before the eye appears in IR imagery where the cloud field is dominated by periodic, repeating cloud structures-which we henceforth identify as the tilt-modulated convective asymmetry (TCA) - which are localized modulations on top of the central dense overcast (CDO) and diurnal cycle. These features appear much larger in terms of spatial expanse than what one would expect of a typical small-scale convective "burst" (e.g., Didlake and Houze 2013; Guimond et al. 2016). They also appear with a frequency much slower than a short-lived "burst" (e.g., Didlake and Houze 2013; Guimond et al. 2016), where the "burst" exists on time scales of tens of minutes. The TCAs, defined within a region of cloud tops colder than $-70^{\circ} \mathrm{C}$, pulse in a range of $4-8 \mathrm{~h}$ with a scale of approximately $10000 \mathrm{~km}^{3}$ or $5 \%$ the size of the total time-averaged cloud shield defined herein. The TCAs discussed in this manuscript occur almost exclusively on the left-of-shear portion of the TC core, do not rotate, and, as discussed by Ryglicki et al. (2018, hereafter Part II), have a specific cause: they are modulated by the nutation of the tilt of the vortex. Here, "nutation" is defined as a higher-order oscillation superposed on the precession of the tilt of the vortex.

As Part I of this series, this manuscript outlines the "atypical" pathway to RI and then focuses on the satellite observations and large-scale model analyses and reanalyses to determine distinguishing characteristics of these unique and atypical TCs. This work will describe binding physical parameters that can serve to constrain idealized modeling simulations, as well as a number of features necessary for corresponding model "verification." Our 
goal is to differentiate thoroughly how this group of atypical TCs differs from so-called "classic" RI TCs (Knaff 2008; Kaplan et al. 2015) and to provide information on RI precursors that can be deduced from satellite and model analyses. Some of the kinematic details of TCAs are explored in Part II, though we can briefly state that these features are collections of buoyant convective towers localized by the vertical structure of a $\mathrm{TC}$ in shear and enhanced by the nutations on the longer tilt precession. In addition to the TCAs, other specific features that occur prior to RI include migration of the cloud shield upshear, a pronounced asymmetry in farfield WV imagery, upshear WV arcs, and downshear-left outflow jets, features that will be explored more thoroughly in future manuscripts. In "classic" TCs, most notably, these arcs do not exist, and this upshear-downshear asymmetry is not as pronounced.

This study makes use of IR and WV satellite imagery, synoptic reanalysis data, and a TC statistical prediction archive to investigate the specific class of TCs that unexpectedly undergo RI in moderate vertical wind shear. The following subsection lays out the full scenario for this atypical pathway to RI. Section 2 describes the data and methods. Section 3 discusses the historical evolution and the synoptic situation of the TCs, as well as a brief climatological inspection relative to SHIPS-RII. Section 4 contains satellite analysis, first focusing on the evolution of Hurricane Hernan in IR imagery. Using Hernan as a prototypical example, we demonstrate similar behavior among the six TCs. Subsequently, we show additional characteristics of the TCs, including arcs in WV satellite imagery. Concurrent to the analyses of the "atypical" RI TCs, we will compare these TCs with examples of "classic" RI cases. Section 5 provides a discussion about operational considerations and justification for the naming of the new feature, TCA. Section 6 summarizes the manuscript.

\section{b. Overview of the "atypical" pathway}

Figure 1 is a schematic of the atypical process, highlighting necessary environmental wind conditions for its existence ("A") and the structural characteristics of TCs associated with it ("B-E"). For this atypical pathway to even be possible ("A"), the strongest environmental winds must be concentrated high in the troposphere. This fact is not altogether unique, as Elsberry and Jeffries (1996) and Finocchio et al. (2016) both have made this observation, but the important details are contained in why this vertical profile matters. Where this profile becomes relevant for these TCs is in how these TCs respond to it ("B"). In these atypical RI cases, the midlevel and upper-level centers rotate out of phase with each other, and prior to RI, the midlevel center migrates farther into the upshear semicircle than its upper-level counterpart (see Part II). As a result of

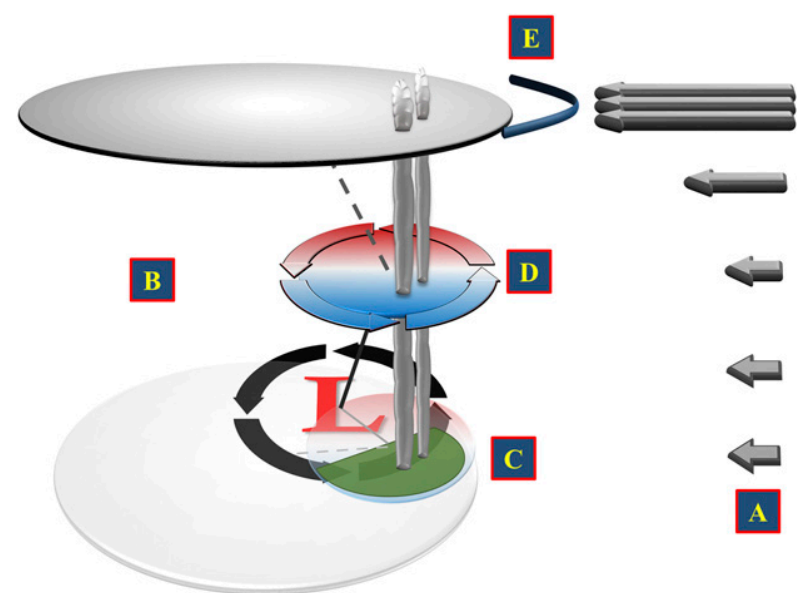

FIG. 1. Schematic of the structure of a TC in the midst of the atypical RI process. Thick arrows are environmental winds; flat arrows are TC winds. A necessary precondition of the environmental flow ("A") is that its strongest flow is confined to the top of the troposphere. The TC then responds to this forcing by tilting. During the realignment process, the TC tilts out of phase with itself, associated with higher-order tilt nutations ("B"). The lowlevel convection migrates into the upshear-left quadrant underneath the midlevel center ("C"). As a result of the tilt and thermal wind balance, there is a vortex-deep cold anomaly associated with the tilt ("D"). Individual convective cells that rise under and into the tilt are themselves buoyant. At the top of the TC, crescent-shaped arc clouds are emitted upshear ("E"; blue coloring is meant to mimic the WV imagery color scale described later). Transparent images over the low-level center ("L") are projections of the mid- and upper levels at the surface. The green semicircle indicates precipitation. Solid black line originating from the $\mathrm{L}$ and the dashed line originating from the midlevel circulation signify the tilt. The gray lines (solid and dashed) over the $\mathrm{L}$ are projections of the tilt on the surface.

the evolution of the midlevel center, convection is localized underneath this tilt ("C"). In addition to this movement, the tilt of the midlevel vortex causes a structural change in the thermodynamic profile of the vortex ("D"). As a result of a tilting vortex, there is a thermal wind balance-induced cold anomaly downtilt (Jones 1995); thus, all convective towers that grow under and into this anomaly while the TC is tilted are buoyant. In addition to the movement of the tilt, crescent-shaped arc clouds become evident in water vapor imagery ("E"). Eventually, RI occurs.

While upshear movement of convection is not a unique idea for intensification of sheared TCs (Stevenson et al. 2014; Rogers et al. 2015, 2016; Zawislak et al. 2016), this atypical pathway directly ties that movement to the tilt. Additionally, it should be noted that this pathway is also different from the downshear reformation theory (Molinari et al. 2006; Molinari and Vollaro 2010; Nguyen and Molinari 2015) since there is no singular convective cell (mesovortex) that becomes the new low-level center. 

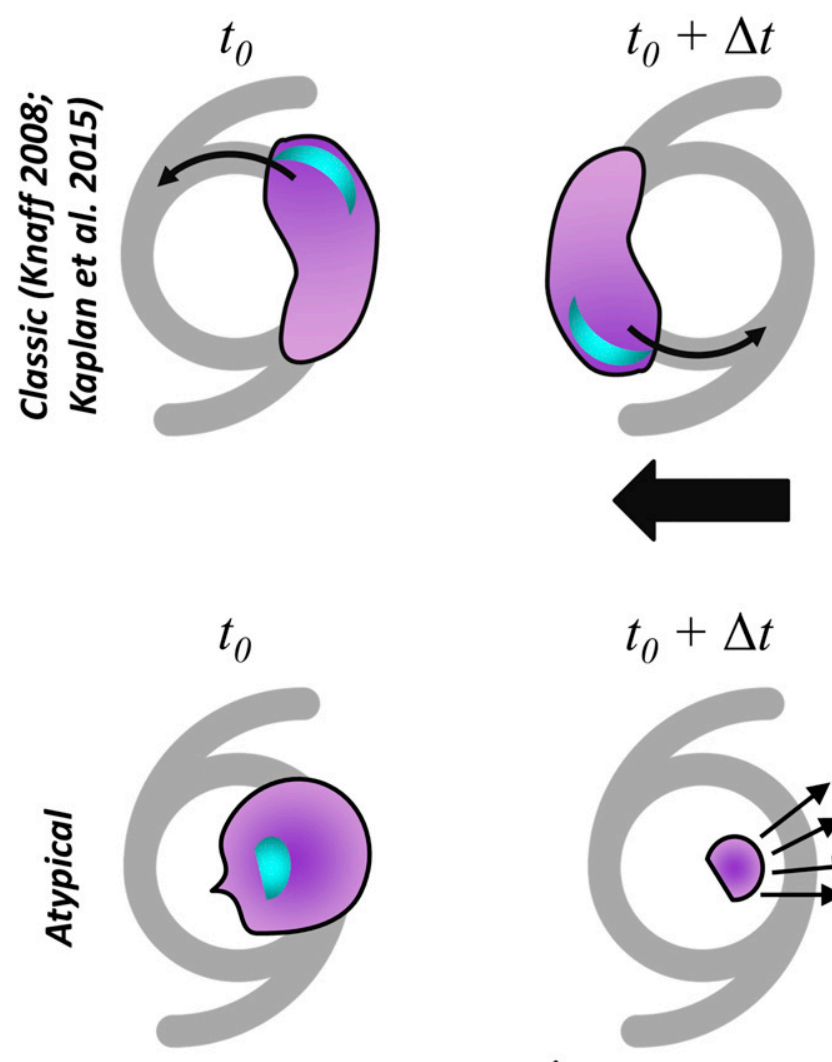

$-70^{\circ} \mathrm{C}$

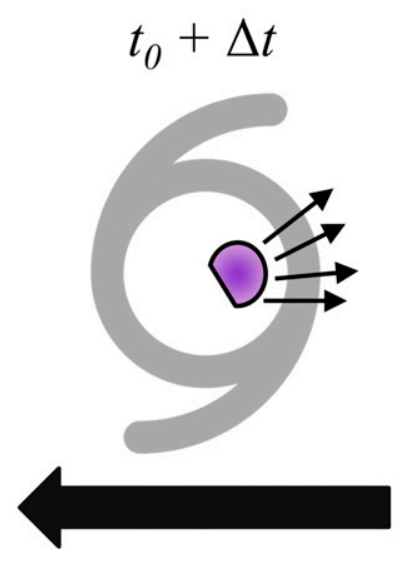

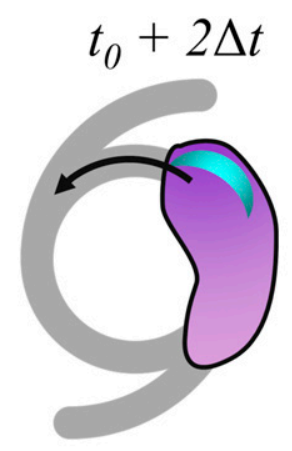

(No upshear $\operatorname{arc~in~WV)~}$

(No upshear warming)

FIG. 2. Schematic comparing the differences in structures in IR satellite imagery associated with (top) "classic" RI TCs with structures identified by Knaff (2008) and used by Kaplan et al. (2015) and (bottom) structures discussed in this article associated with "atypical" RI TCs. Colors are associated with brightness temperatures $\left({ }^{\circ} \mathrm{C}\right)$. Large black arrows are shear vectors. Smaller black arrows indicate either (top) rotation or (bottom) upshear expansion. Included in the atypical evolution schematic are two additional features in WV imagery: the upshear arc (in blue, to match the WV color scheme) and warming/drying beyond that (indicated by the orange crescent and the "W"). The time scale $(\Delta t)$ is approximately $2-4 \mathrm{~h}$.

This process is complex. It also incorporates some newer ideas about intensity evolution in shear, such as out-ofphase tilt oscillations' modulating convection. As a result, we are going to split its analysis into several parts. For this particular manuscript, we focus primarily on what is observable. Generally, IR imagery is useful for determining the movement of the midlevel vortex. An important finding is that the dynamic evolution of the midlevel tilt can be abstracted from IR satellite imagery (see Part II for more details). Figure 2 is a schematic comparing "classic" pre-RI IR patterns identified by Knaff (2008) and used by SHIPSRII (Kaplan et al. 2015) and the "atypical" pre-RI IR patterns to be discussed in this manuscript along with WV characteristics. The fundamental difference is near-core rotation (classic) as opposed to upshear expansion (atypical). In addition, in WV imagery, the episodic pulsing causes upshear arcs and the far-field warming/drying. As observational examples, Fig. 3 depicts a comparison of "classic" 2009 EPAC Rick and the prototypical "atypical" case, 2008 EPAC Hernan, both oriented with the shear vector pointing to the left over the span of approximately $5 \mathrm{~h}$. The large, cold cloud mass circumnavigates Rick's core, while the cloud mass of Hernan pulses over the core on the left-of-shear side, expanding upshear. Both are discussed in more detail later in the manuscript. Both also subsequently became major hurricanes. Operational forecasts of Rick's RI were successful in that RI was forecast by official forecasters; for Hernan, they were not (further discussed in sections 3 and 4). While we cannot say for sure what causes these convective blow ups in Rick, we do know for a fact that they are associated with the tilt in Hernan (see Part II). What separates this feature from traditional interpretations of the $\mathrm{CDO}$ is that the modulated cloud cover is $-70^{\circ} \mathrm{C}$ or colder, exists on top of the $\mathrm{CDO}$, and also pulses with a periodicity faster than that of the diurnal cycle (to be discussed in section 4). 

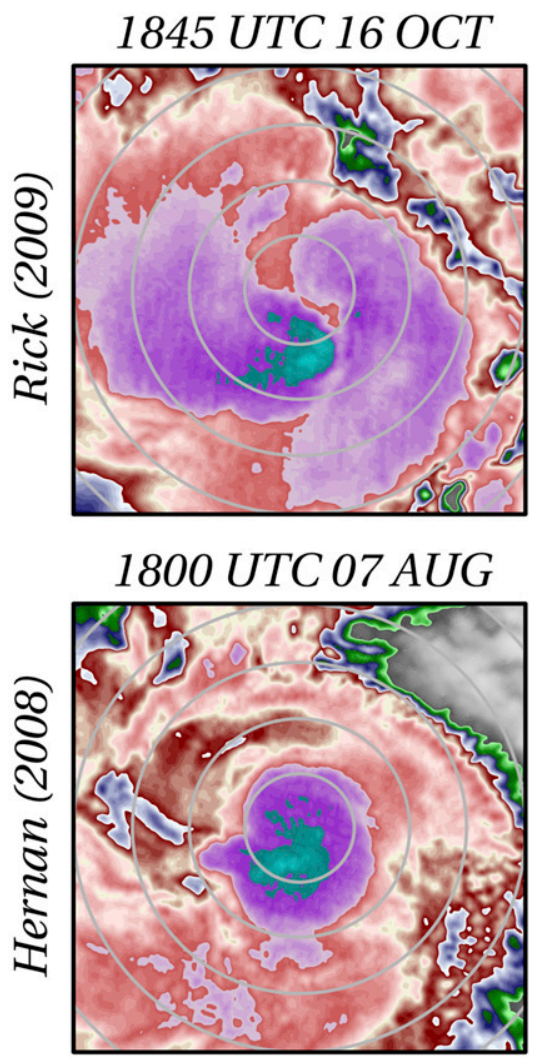

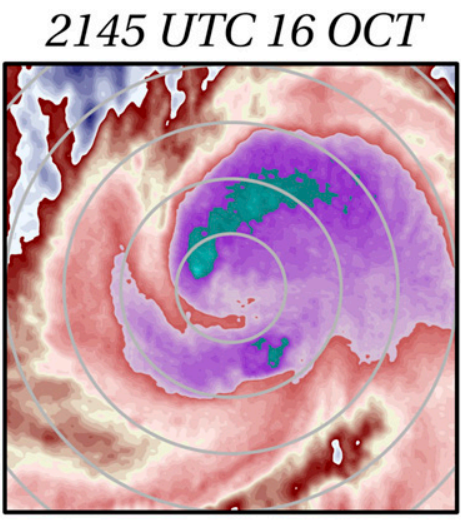

2100 UTC 07 AUG

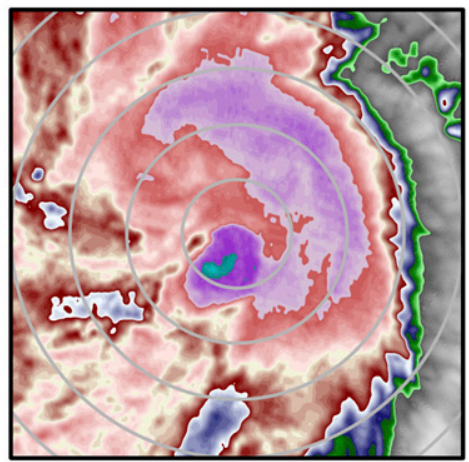

2345 UTC 16 OCT

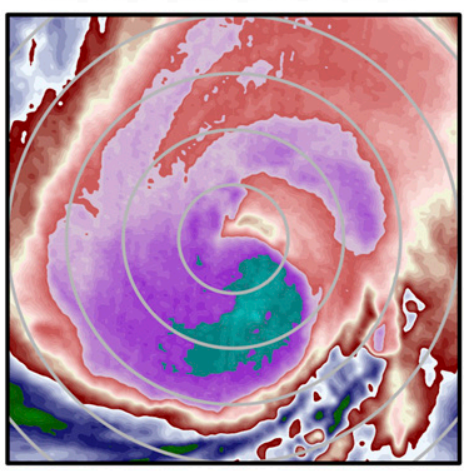

2300 UTC 07 AUG

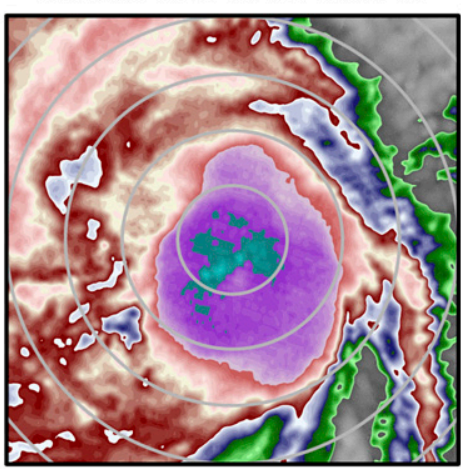

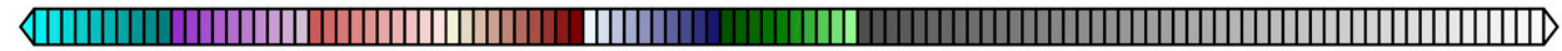

$\begin{array}{lllllllllll}-90 & -80 & -70 & -60 & -50 & -40 & -30 & -20 & -10 & 0 & 10\end{array}$

FIG. 3. IR satellite $\left({ }^{\circ} \mathrm{C}\right.$ ) comparison of (top) 2009 EPAC Hurricane Rick and (bottom) 2008 EPAC Hurricane Hernan, illustrating observations as described in Fig. 2. Shear vector in both cases is pointing left. Gray circles are radii every $50 \mathrm{~km}$.

\section{Data and methods}

\section{a. Data}

Geostationary satellite observations of a total of $10 \mathrm{TCs}$ are analyzed: six "atypical" TCs that undergo RI in moderate vertical wind shear (Guillermo, Hernan, Norbert, Fabio, Hilda, and Joaquin) and four "classic" TCs that undergo RI in light vertical wind shear (2005 NATL Wilma, 2009 EPAC Rick, 2012 EPAC Emilia, and 2015 EPAC Patricia). They are cataloged in Table 1. The first six provide an ideal description of the characteristics of this "atypical" RI in moderate vertical wind shear. The second group all fit Knaff's (2008) constraints. The time frame of the TCs covered makes use of data from six Geostationary Operational Environmental Satellites (GOES): GOES-8, GOES-9, GOES-11, GOES-12, GOES-13, and GOES-15. The temporal resolution of all of the storms for the geostationary satellite data is $30 \mathrm{~min}$, except for Joaquin, which is $15 \mathrm{~min}$, since it was located in an area with a higher scan frequency. This also limits our observations to those basins nearest to the continental United States.
The focus of this study will be on the IR $(10.7 \mu \mathrm{m})$ and WV (6.5 or $6.7 \mu \mathrm{m}$, depending on specific satellite) channels. The geostationary data are interpolated to storm-following polar coordinates. The bicubic-splineinterpolated best track (HURDAT2; Landsea and Franklin 2013) position is used for the center. The data are then interpolated to a polar grid using Delaunay triangulation (Delaunay 1934) outward to a radius of $500 \mathrm{~km}$ with a radial grid spacing of $1 \mathrm{~km}$ and 720 points in the azimuth (i.e., $d \lambda=0.5^{\circ}$ or $\pi / 360$ radians). Time series are then created

TABLE 1. List of "atypical" RI cases and "classic" RI cases examined in this study.

\begin{tabular}{ll}
\hline \multicolumn{1}{c}{ "Atypical” RI } & \multicolumn{1}{c}{ "Classic" RI } \\
\hline 1997 EPAC Guillermo & 2005 NATL Wilma \\
2008 EPAC Hernan & 2009 EPAC Rick \\
2008 EPAC Norbert & 2012 EPAC Emilia \\
2012 EPAC Fabio & 2015 EPAC Patricia \\
2015 EPAC Hilda & \\
2015 NATL Joaquin & \\
\hline
\end{tabular}



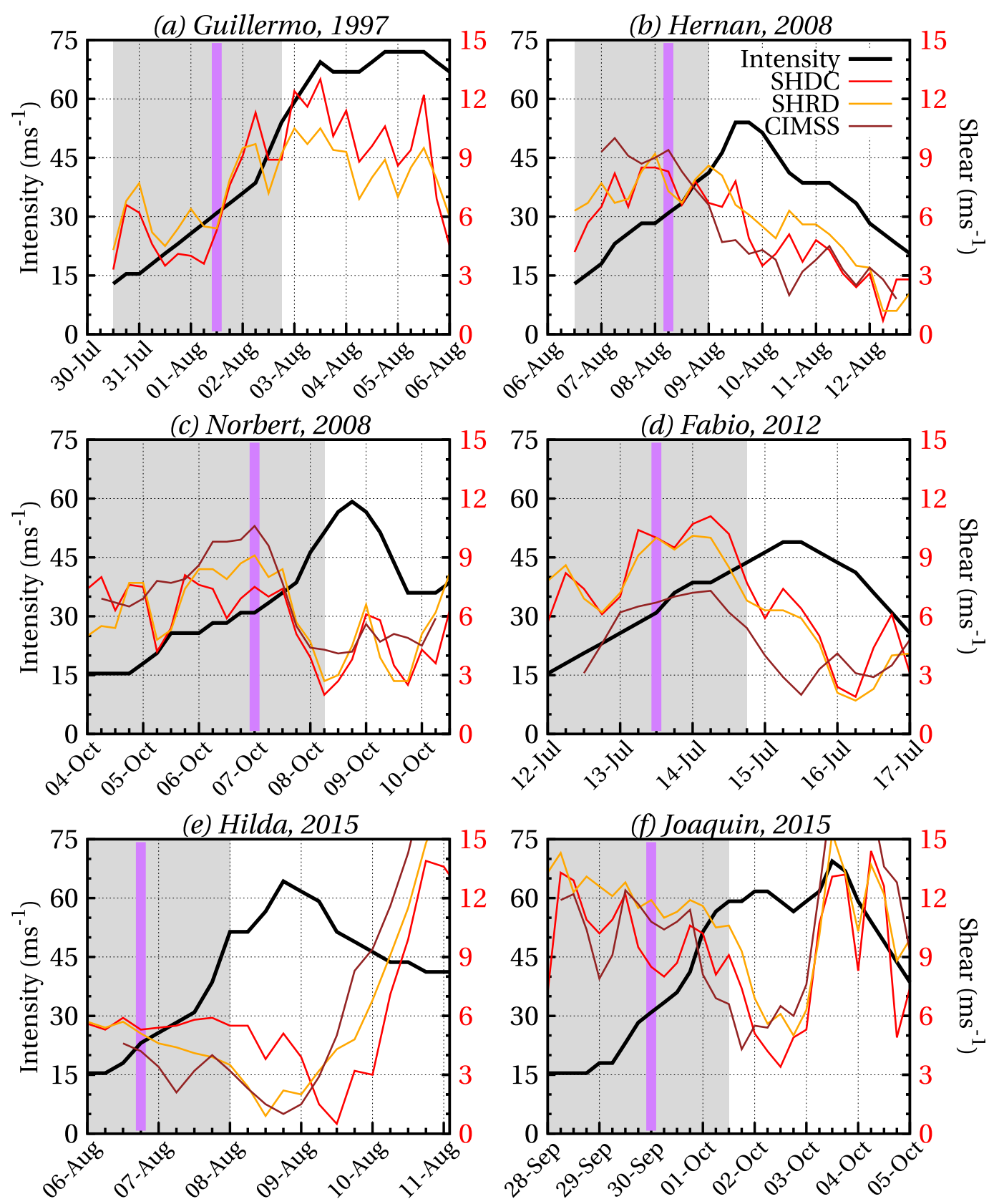

FIG. 4. Time series of best track intensities (black line; left $y$ axis), the two SHIPS shear calculations (red and orange lines, respectively, for SHDC and SHRD; right $y$ axis; see text for definitions), and the CIMSS shear calculation (brown line; right axis) for (a) Guillermo, (b) Hernan, (c) Norbert, (d) Fabio, (e) Hilda, and (f) Joaquin. Gray boxes highlight the time frame from genesis to eye appearance. Purple lines are onsets (or approximation, for Fabio) of RI.

using these storm-relative data. The details of the signal processing tools used for these analyses are presented in the appendix.

For the large-scale synoptic forcings, various databases are used. To diagnose vertical wind shear, the SHIPS database is used. Despite some limitations in its shear methodology (Gallina and Velden 2000; Gallina 2002; Gallina and Velden 2002; Velden and Sears 2014),
SHIPS is used since it is a robust database that incorporates many relevant TC-related metrics and has readily available climatological studies (Kaplan et al. 2010, 2015; Rozoff et al. 2015). The two 200-850-hPa shear calculations are explored. The first vertical wind shear metric (SHDC) removes the divergent component of the vortex and then calculates the shear in a $500-\mathrm{km}$ circle surrounding the storm. The second (SHRD) does not remove 
TABLE 2. Means and standard deviations for 200-850-hPa shear (SHDC; $\mathrm{m} \mathrm{s}^{-1}$ ), low-level relative humidity (RHLO; \%), 200-hPa divergence (D200; $10^{-7} \mathrm{~s}^{-1}$ ), and difference between current winds and maximum potential intensity (POT; $\mathrm{m} \mathrm{s}^{-1}$ ) from Kaplan et al. (2010).

\begin{tabular}{lcc}
\hline \hline Parameter & North Atlantic & Eastern Pacific \\
\hline SHDC & $5.1,2.0$ & $3.9,1.5$ \\
RHLO & $73.5,6.2$ & $78.4,4.8$ \\
D200 & $49.2,30.0$ & $59.1,30.6$ \\
POT & $39.7,11.3$ & $49.2,9.4$ \\
\hline
\end{tabular}

the vortex and calculates shear in an annulus between 200 and $800 \mathrm{~km}$ radially from the center. As an additional corroboration and comparison, we include the Cooperative Institute for Meteorological Satellite Studies (CIMSS) satellite wind-derived shear calculations (Velden and Sears 2014) where available (Guillermo is unavailable). The primary difference between the SHIPS calculations and the CIMSS calculations is that in SHIPS, the shear calculation is the difference between two levels (200 and $850 \mathrm{hPa}$ ), while in CIMSS, they use layer averages (150300 and $700-925 \mathrm{hPa}$ ). For track and intensity, the best track dataset is used. The daily mean SSTs are obtained from the $1^{\circ}$ optimally interpolated SST version 2 (OISSTv2) dataset (Reynolds et al. 2002). For synoptic characterizations, the $\sim 0.75^{\circ}$ ERA-Interim dataset (Dee et al. 2011) is used. When analyzing storm-relative winds, the fields are first smoothed with a Shuman (1957) filter 10 times and then averaged in a $5^{\circ} \times 5^{\circ}$ box surrounding the best track center.

\section{b. Storm size}

In section 4, satellite analyses are presented using radially dependent observations. Each of the six "atypical" RI TCs has a different size in terms of total cloud coverage; therefore, structural information in an annulus surrounding a very small storm may not provide the same information as the same annulus for a very large storm. To better characterize storm size and justify variations in the annuli, the radial extent of the timeaveraged, storm-relative symmetric $-30^{\circ} \mathrm{C}$ contour is used as a simple quantification, based on guidance from Knaff et al. (2014) and SHIPS-RII (Kaplan et al. 2015). This analysis is not meant to provide definitive guidance on TC size or on the variability thereof, but it is a practical means for quantifying relative size among the TCs. The storm-centered IR brightness temperatures are averaged over the life cycles of each of the TCs, except for Guillermo, which is truncated to the first 8 days of its life cycle, given the relative insignificance of its later life cycle to this evolution (its total best track entry is 24 days).

\section{Large-scale storm characteristics}

The overall motivation for this study of the six specific TCs is the atypical nature of their intensification evolutions. For example, the RIs of Hernan (Knabb 2008), Norbert (Brown 2008), Hilda (Beven 2015a), and Joaquin (Beven 2015b; Brown 2015) all were unexpected by the official forecasters, mainly due to computed $200-850-\mathrm{hPa}$ shear values and presentations on satellite. Fabio continued to intensify in vertical wind shear values, which, according to Kaplan et al. (2010), should have weakened the TC or at least prevented its continual intensification (Berg 2012). Figure 4 summarizes the intensity trends and the shear values from SHRD, SHDC, and CIMSS for all six of the TCs discussed here, where the gray boxes signify the time frames for each TC from genesis to the appearance of the eye in IR imagery. Over the entirety of the gray boxes, despite some storm-to-storm variability, the average vertical wind shear is $7.32 \mathrm{~m} \mathrm{~s}^{-1}$ for SHDC, $7.79 \mathrm{~m} \mathrm{~s}^{-1}$ for SHRD, and $7.31 \mathrm{~m} \mathrm{~s}^{-1}$ for CIMSS, while the average of all 17 time series is approximately $7.5 \mathrm{~m} \mathrm{~s}^{-1}$.

When deriving their RI index, Kaplan et al. (2010) identified seven predictors for RI coincident across both EPAC and NATL. Follow-on studies have also altered predictors and refined the numbers (Kaplan et al. 2015; Rozoff et al. 2015). To simplify and streamline the synoptic discussion, we will focus on the four "large scale" predictors from the three studies: $850-700-\mathrm{hPa}$ relative humidity (RHLO), 200-hPa divergence (D200), 200-850 hPa shear (SHDC), and the difference between maximum potential intensity (MPI; Rotunno and Emanuel 1987) and current intensity (POT). The potential intensity difference allows for a simple quantification of thermodynamic favorability. It should be noted that some of the refined predictors, such as total precipitable water and ocean heat content, do not cover the entire historical dataset in SHIPS (including Guillermo), so homogeneous climatological comparisons cannot be made with the newer predictors at this time. Lower values of shear and higher values of relative humidity, upper-level divergence, and potential intensity are more favorable for TC development (Kaplan et al. 2010). While not necessarily causative, this dataset does allow for a way to quantify a portion of the parameter space of these TCs. The means and standard deviations of the four parameters from Kaplan et al. (2010) are in Table 2. Figure 5 is a collection of the intensification time series of the six TCs now with the four parameters centered about their climatological RI means and normalized by their standard deviations (Kaplan et al. 2010).

The key takeaways from Fig. 5 are fourfold. First, with the exception of a period of time early in Guillermo's life cycle (Fig. 5a), the shear ranges between one and five standard deviations higher than climatological values. 

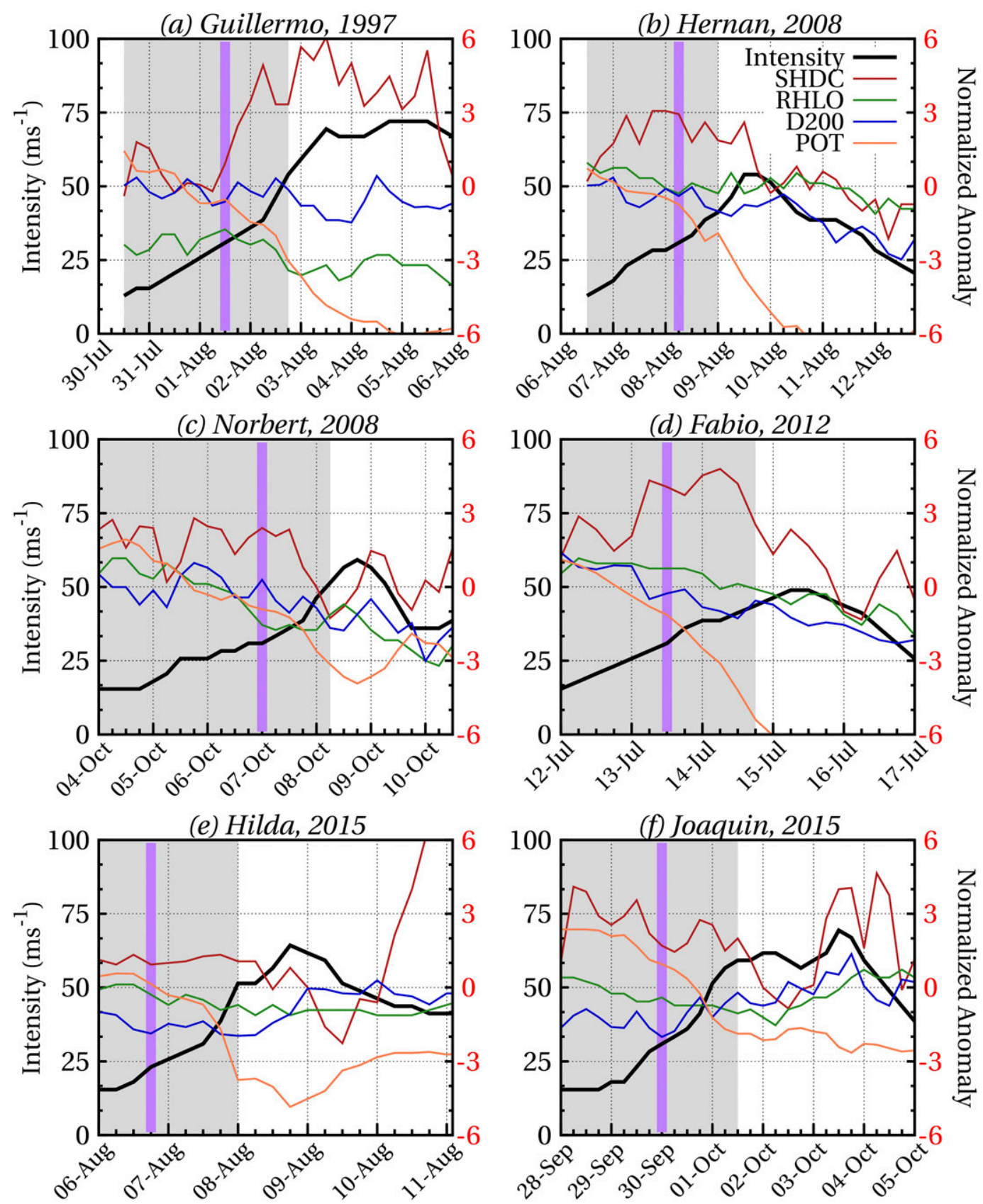

FIG. 5. Time series of (left $y$ axis) best track intensities (black line) and (right $y$ axis) SHIPS-RI normalized anomalies of SHDC (red line), RHLO (green line), D200 (blue line), and POT (coral line) for the six TCs. See text for definitions. Gray boxes are as in Fig. 1. Purple lines are as in Fig. 1. Anomalies are centered and normalized by values by basin given in Table 1 .

Second, with the exception of Guillermo, low-level humidity is generally within one standard deviation of the climatological RI mean, implying that these TCs were not in excessively moist environments relative to other TCs that undergo RI. Third, the upper-level divergence is consistently smaller, in a normalized anomaly sense, than the shear. Fourth, with the exception of Joaquin, the potential intensity is generally within climatologically expected values (SSTs are generally around $28^{\circ} \mathrm{C}$, except for Joaquin, whose underlying SSTs are around $30^{\circ} \mathrm{C}$ ). To illustrate the importance of these relationships and how they compare with "classic" RI TCs, four null cases are selected for comparison: 2005 NATL Wilma, 2009 EPAC Rick, 2012 EPAC Emilia, and 2015 EPAC Patricia (Figs. 6a-d). In the 

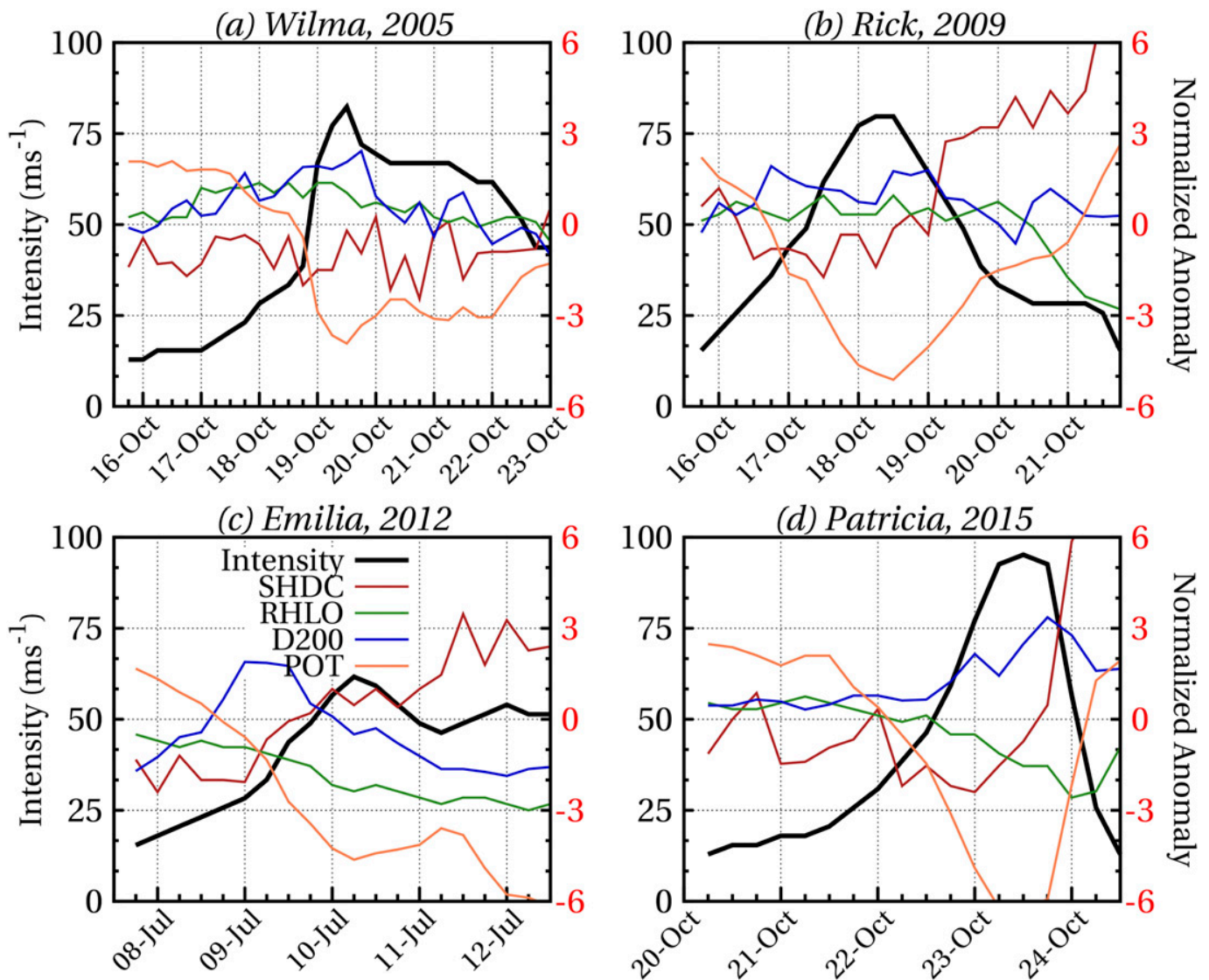

FIG. 6. As in Fig. 5, but for the "classic" cases: (a) 2005 NATL Wilma, (b) 2009 EPAC Rick, (c) 2012 EPAC Emilia, and (d) 2015 EPAC Patricia.

four "classic" RI TCs-all of which contain the feature identified by Knaff (2008) - shear is generally at or well below the climatological mean. Low-level relative humidity is generally within one standard deviation of the climatological mean. The potential intensity begins two (or more) standard deviations higher than climatology. The upperlevel divergence is consistently higher than the shear. From Figs. 5a-f, we deduce that for these atypical and marginal cases, shear is consistently larger than upper-level divergence, often by two to three standard deviations. Here, we can state explicitly that the "unfavorable" condition for $\mathrm{RI}$ is shear, which is at least one standard deviation greater than RI climatology (Kaplan et al. 2010). This relationship is the opposite for the classic cases. Operationally, the forecasts of RI for Wilma (Franklin 2005), Rick (Blake 2009), Emilia (Blake 2012), and Patricia (Pasch 2015) were all well communicated and successful in a binary sense, where even though the exact intensification rate might not have been forecast, the idea that RI would occur was explicitly mentioned in the discussion.

Synoptically, all six of the TCs share an environmental feature in common: the upper-level shearing mechanism. Figure 7 is a collection of the $200-\mathrm{hPa}$ winds from ERA-Interim for the six TCs prior to each undergoing RI. All six were under the influence of a large upper-level anticyclone. It should be noted here that these anticyclones are not displaced anticyclones from the TCs themselves (Wu and Emanuel 1993) but are preexisting, synoptic-scale anticyclones. The physical significance of the anticyclones is the vertical structure of the environmental winds. As was shown by Hoskins et al. (1985) and Wirth (2001), upper-level anticyclonic PV anomalies are much shallower in the vertical when compared with their cyclonic counterparts, given the same thermal anomaly magnitude and the same radial size. Figure 8 is a slight modification of Fig. 15 from Hoskins et al. (1985), comparing an idealized upper-level cyclonic PV anomaly (Fig. 8a) with an idealized upper-level anticyclonic PV anomaly (Fig. 8b) of the same radial size and thermal magnitude $(24 \mathrm{~K})$. Notice where the $15 \mathrm{~m} \mathrm{~s}^{-1}$ wind contour is between the two: from the cyclone, its lowest extent is $800 \mathrm{hPa}$, while from the anticyclone, its lowest extent is $300 \mathrm{hPa}$. To illustrate the difference between 
(a) Guillermo, 00 UTC 02 AUG

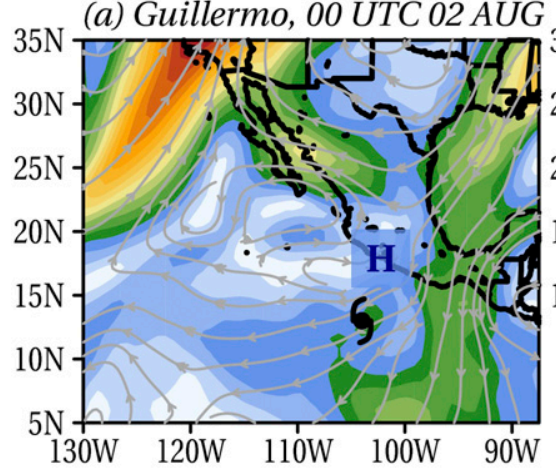

(d) Fabio, 12 UTC $13 \mathrm{JUL}$

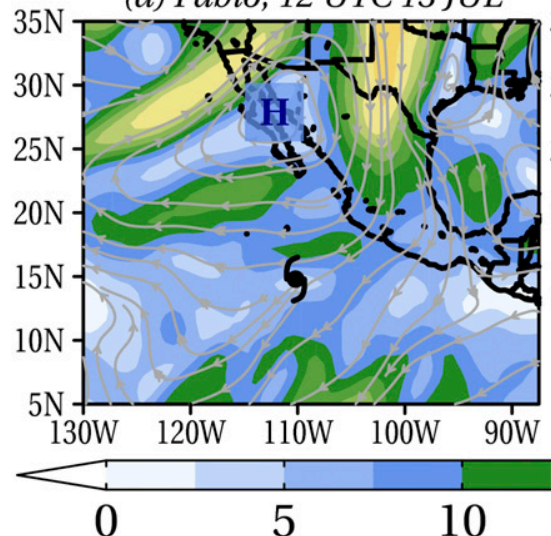

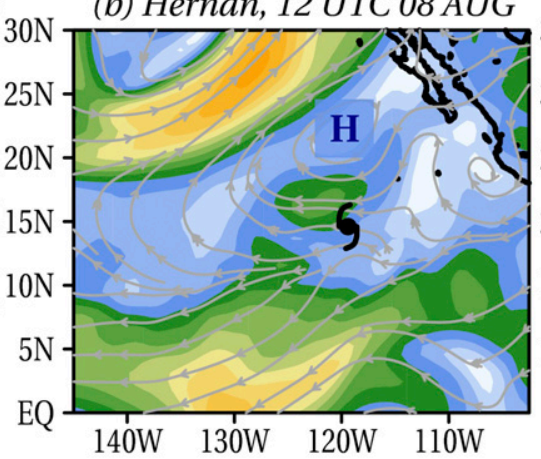

(e) Hilda, 12 UTC 07 AUG

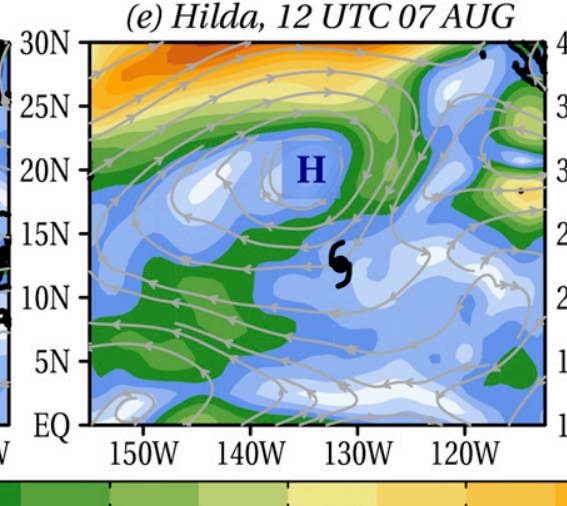

130W 120W 110W 100W 90W

(f) Joaquin, 06 UTC 30 SEP

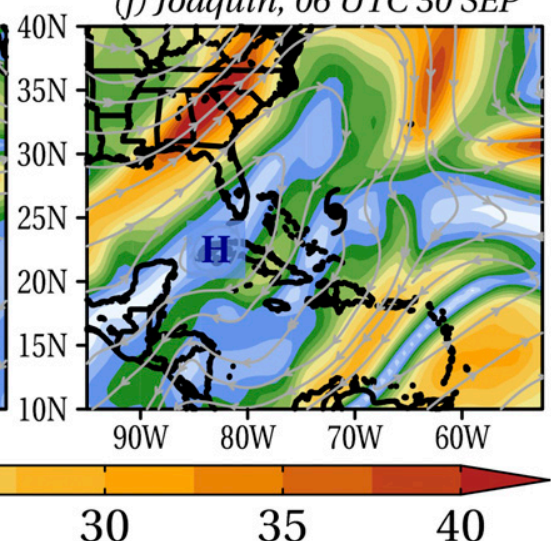

FIG. 7. The 200-hPa wind magnitudes $\left(\mathrm{m} \mathrm{s}^{-1}\right.$ ) and streamlines from ERA-Interim at times prior to RIs of (a) Guillermo, (b) Hernan, (c) Norbert, (d) Fabio, (e) Hilda, and (f) Joaquin. The blue "H" symbols signify the location of the upper-level anticyclone; the black TC symbols indicate the location of the TC.

anticyclonic and cyclonic upper-level anomalies in reality, Fig. 9 presents an example of each during Joaquin's existence since Joaquin was the only one of the TCs to experience shear from both an upper-level anticyclone and an upper-level trough in relatively quick succession. Additionally, the shear values from both phenomena are approximately three standard deviations larger than the SHIPS RI climatology (Fig. 5f), but the vertical structure is different. The anticyclone's strongest winds are confined to higher levels in the troposphere, fulfilling the necessary environmental conditions described in section $1 \mathrm{~b}$, while the trough's winds extend more deeply into the troposphere, a fact that some studies (e.g., Elsberry and Jeffries 1996; Velden and Sears 2014; Finocchio et al. 2016) indicate is more detrimental to TC development.

\section{Satellite analyses}

This section describes the IR and the WV analyses corresponding with the "atypical" and "classic" RI cases. The main feature in the IR imagery is the presence of periodic convection on a 4-8-h time scale and a characteristic shape of the cloud field that exists as a modulation above the CDO. The main features in the WV imagery are crescent-shaped clouds that exist upshear beyond the CDO separated by a thin warm gap and a warm/cold upshear/downshear dichotomy in WV brightness temperatures. In addition, we highlight both IR and WV imagery of one case (Joaquin) to explore the radial propagation speeds of the boundaries of the TCAs and the arcs discussed in this section.

\section{a. Infrared imagery}

Figure 10 illustrates the time-averaged symmetric brightness temperatures of each of the "atypical" RI TCs, from which we estimate size. Guillermo is the largest by this metric, while Hilda is the smallest-roughly $17 \%$ the size of Guillermo in terms of areal coverage within the time-averaged $-30^{\circ} \mathrm{C}$ contour. In the following analyses, the spatial distributions of convective variability are discussed, so it is important to recognize that there will be differences in the analyses among the TCs based on their overall sizes. For example, an annular average between $300-$ and $400-\mathrm{km}$ radius may not contain the same relevant information on convective activity in 


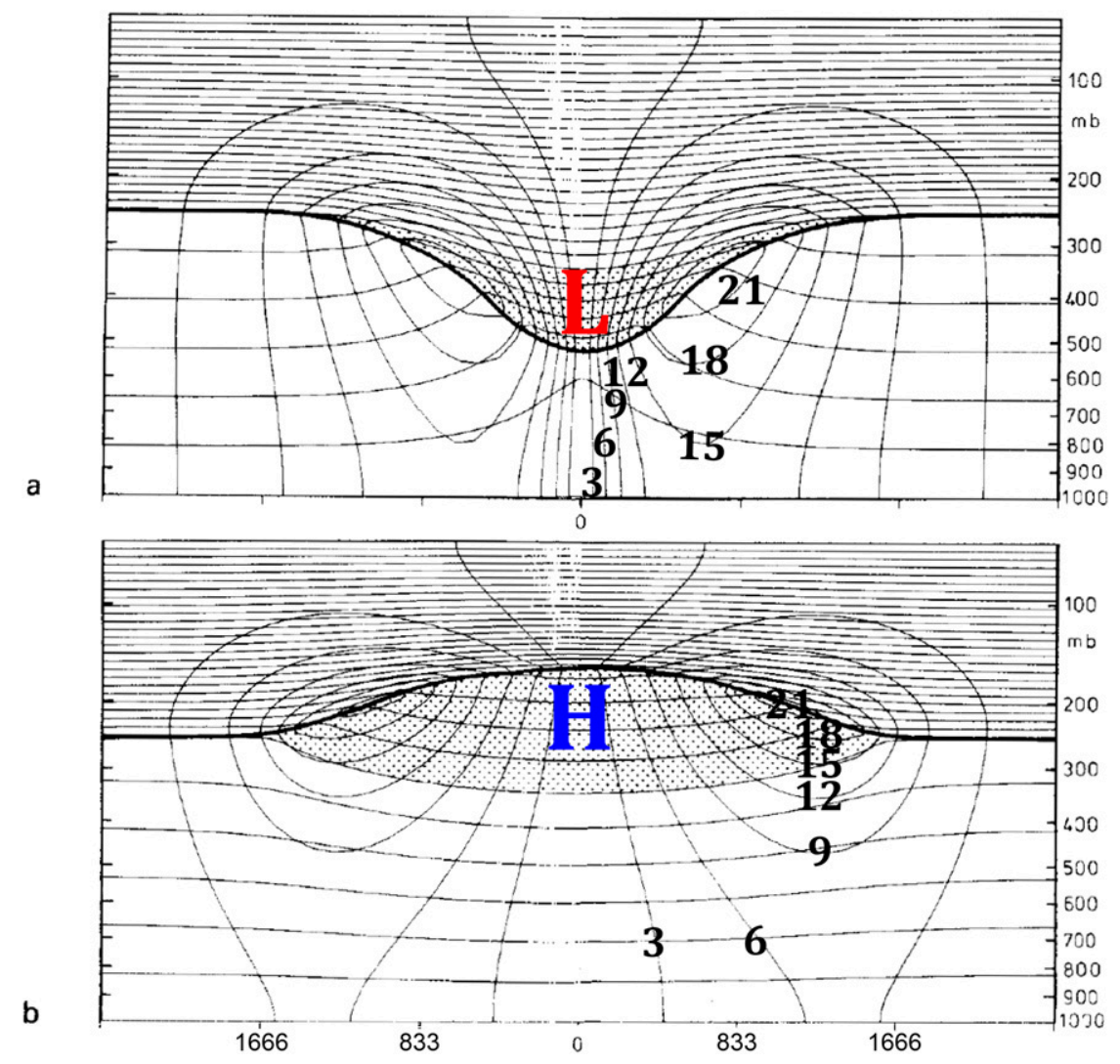

FIG. 8. Adapted from Hoskins et al. (1985): radius-pressure comparison of (a) cyclonic and (b) anticyclonic tropopause PV anomalies. Stippling is the imposed anomaly in both cases, where the temperature anomaly is $\pm 24 \mathrm{~K}$. Thick black line is the tropopause. Labeled contours indicate isotachs $\left(\mathrm{m} \mathrm{s}^{-1}\right)$. The abscissa is radial distance from the center of the anomaly $(\mathrm{km})$. Unlabeled horizontal lines are isentropes.

Hilda as it potentially would in Joaquin; therefore, to provide an accurate depiction of the evolution of the convective variability for each TC, we will adjust the size of the annulus to be centered at approximately half the distance of the time-averaged $-30^{\circ} \mathrm{C}$ contour.

To demonstrate the entire evolution from depression to hurricane in IR imagery for "atypical" RI TCs, Saffir-Simpson hurricane wind scale category 3 major Hurricane Hernan will be used as a prototype. ${ }^{1}$ As indicated in Fig. 4b, Hernan underwent RI despite nearconstant vertical wind shear of $7.5 \mathrm{~m} \mathrm{~s}^{-1}$, moderate shear values that are two standard deviations higher than the RI climatology. As indicated by Fig. 10, Hernan's average radial size is approximately $250 \mathrm{~km}$, and its average areal size is approximately $200000 \mathrm{~km}^{2}$.

\footnotetext{
${ }^{1}$ An animation of Hurricane Hernan's evolution in IR satellite is available in the online supplemental material. We strongly encourage the reader to view this animation before proceeding.
}

Figure 11 highlights a multistep development process of Hernan: the depression phase (Fig. 11a), the upshear shift of the cloud shield (Fig. 11b), an example of corresponding TCAs (framed by the $-70^{\circ} \mathrm{C}$ isotherm in Fig. 11c), and finally the appearance of the eye (Fig. 11d). Within this evolution, the two most prominent features are the shift of the cloud shield and the TCAs.

To clarify the shift of the cloud shield and to illustrate some characteristics of the TCAs, Fig. 12 is a Hovmöller diagram of brightness temperatures around Hernan averaged over an annulus from 80 to $130 \mathrm{~km}$. From genesis (0000 UTC 6 August) to the snapshot in Fig. 11b (1430 UTC 7 August), the coldest cloud temperatures slowly migrate from right of shear to downshear to left of shear, and finally to almost completely upshear. This migration takes place over roughly $34 \mathrm{~h}$. After this, the coldest cloud tops, presumably associated with the strongest convective activity, are localized left of shear. Embedded within the CDO are additional repeating cold-cloud-top minima that occur with an apparent periodicity. To quantify this 

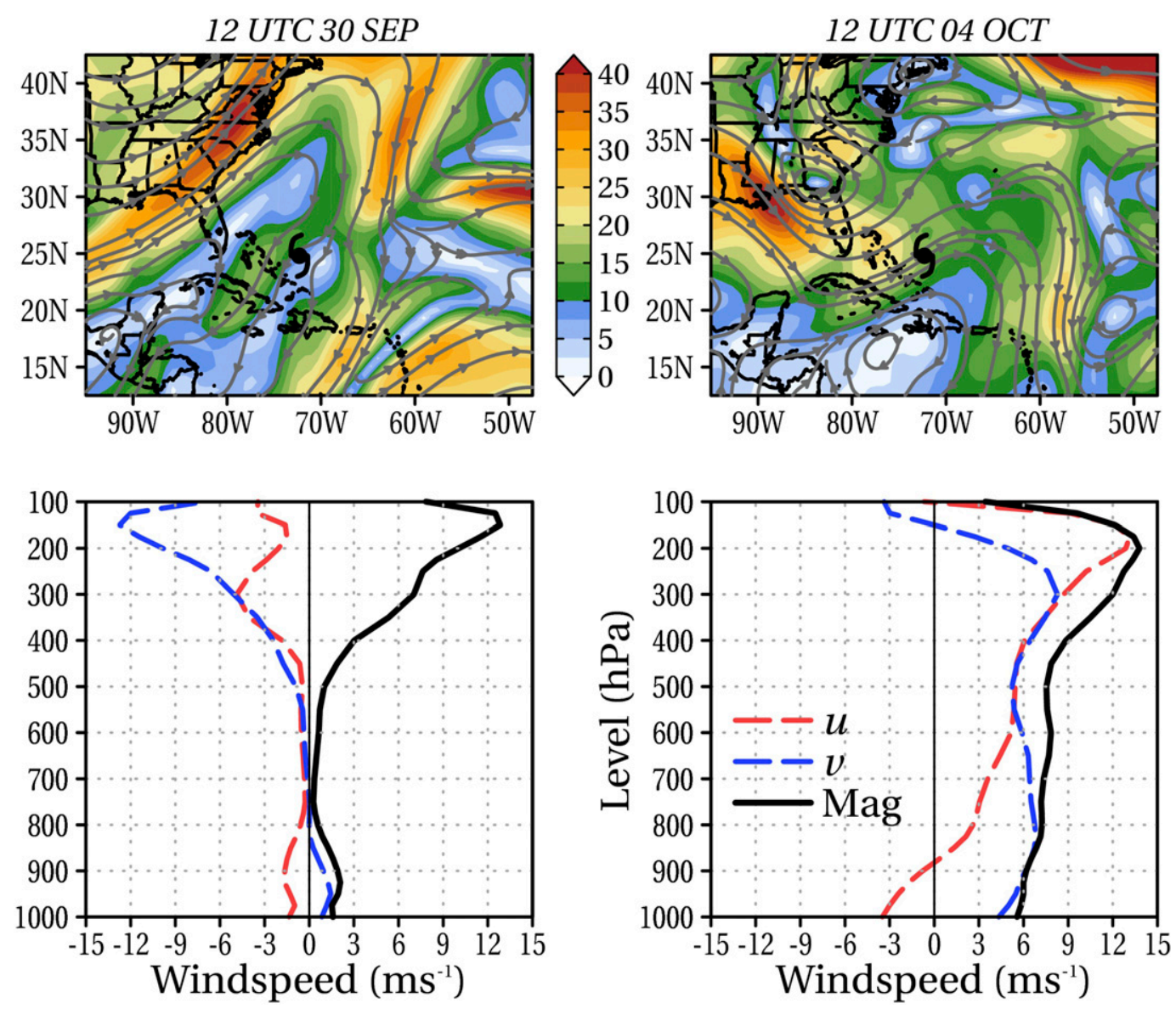

FIG. 9. The (top) 200-hPa winds $\left(\mathrm{m} \mathrm{s}^{-1}\right)$ and (bottom) vertical profile of the winds $\left(\mathrm{m} \mathrm{s}^{-1}\right)$ surrounding 2015 NATL Joaquin at (left) 1200 UTC 30 Sep and (right) 1200 UTC 4 Oct from ERA-Interim. For the vertical profiles, the winds are smoothed with a Shuman filter and then averaged in a $5^{\circ} \times 5^{\circ}$ box around the TC center.

periodicity, we consider all regions colder than $-70^{\circ} \mathrm{C}$ within $200 \mathrm{~km}$ of the TC center over the life cycle of Hernan. Figure 13a shows the time series of this quantity, both unfiltered and filtered, using the methods described in the appendix. The TCAs themselves are responsible for changes between 5000 and $15000 \mathrm{~km}^{2}$ in cold cloud coverage. The detrended time series is next analyzed using wavelet analysis, which indicates that

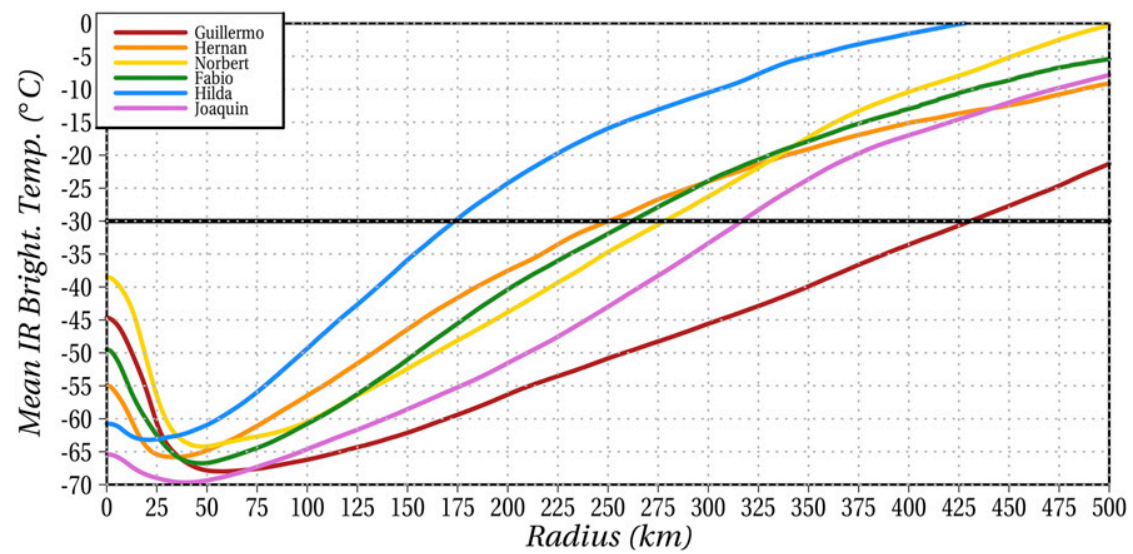

FIG. 10. Time-averaged, symmetric IR brightness temperature $\left({ }^{\circ} \mathrm{C}\right)$ for each of the six TCs. 

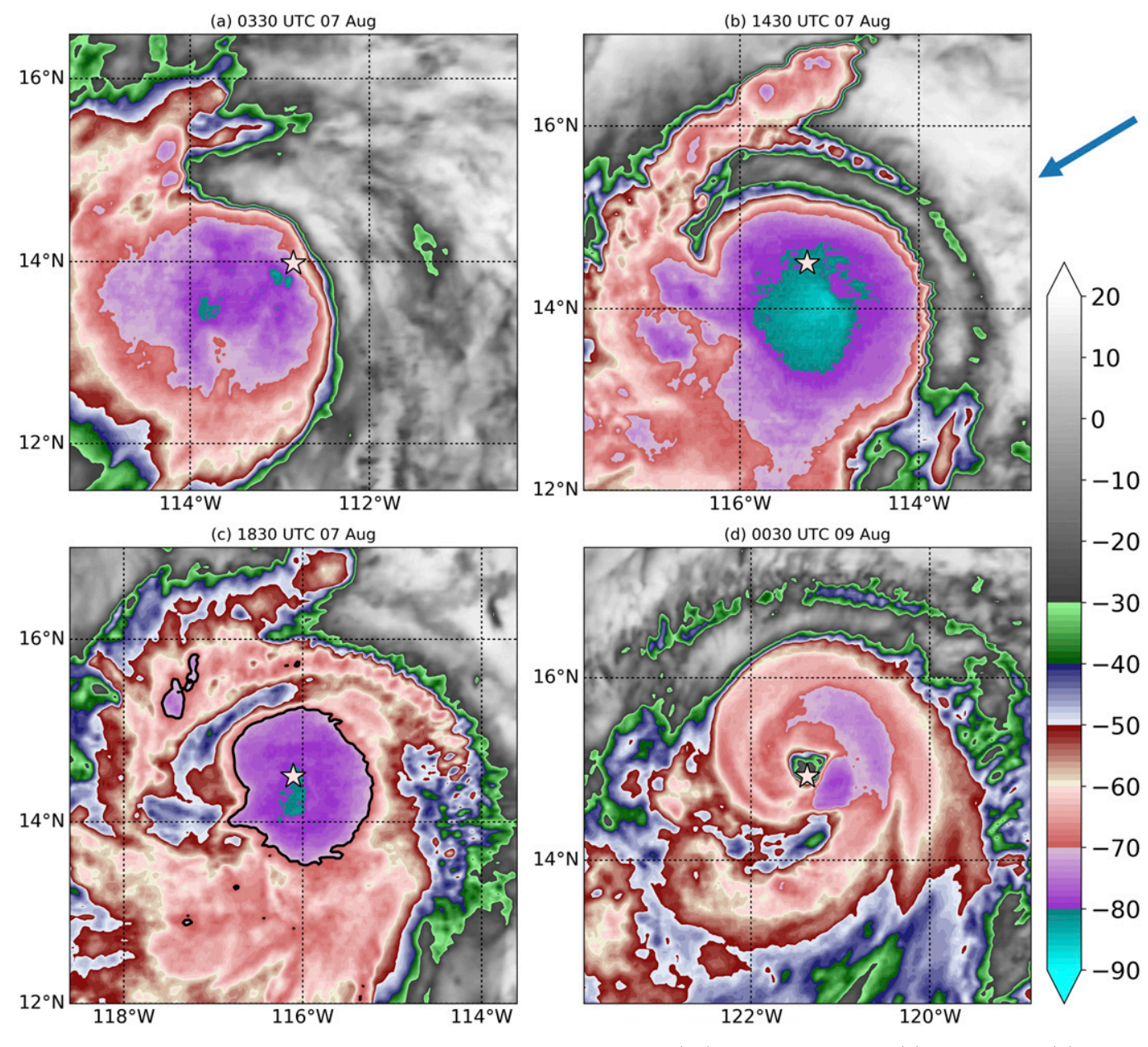

FIG. 11. The four phases of Hurricane Hernan's evolution in IR $\left({ }^{\circ} \mathrm{C}\right)$ satellite imagery: (a) depression, (b) cloud shift, (c) TCA, and (d) eye. Blue arrow is the average shear direction, averaged in time from 0000 UTC 7 Aug to 0000 UTC 9 Aug. Star is the center of the TC. The black outline in (c) is the $-70^{\circ} \mathrm{C}$ contour, which also outlines the TCA surrounding the center.

the TCAs of Hernan appear with a periodicity of approximately $4.25 \mathrm{~h}$ (Fig. 13b). For a further description of the wavelet analysis, see the appendix.

Tying this to the operational situation of Hernan, we cite the excellent discussion that Avila (2008) wrote of Hernan on 8 August 2008:

The cloud pattern has not changed very much during the past several hours and due to shear. ... The low-level center still appears to be located on the edge of the deep convection. Latest microwave data reveal that the low- and mid- level centers are a little more aligned than a few hours ago but most of the deep convection remains south of the center. The outflow is established only in the western semicircle. ... Intensity guidance is not showing any significant intensification. In fact. . . most of the models suggest that Hernan has peaked already. Based on continuity...the official forecast still shows a very modest strengthening during the next 24 hours bringing Hernan to hurricane status. This is becoming less likely to occur since the shear is not relaxing as anticipated.

Avila (2008) specifically mentions the location of the lowlevel center, the vertical alignment derived from microwave imagery, and the (perceived) lack of shear relaxation, all three of which are critical parts of this atypical evolution. Note that as of this forecast, and the several that led up to Knabb's (2008) assessment of the missed RI, Hernan had already begun exhibiting two IR characteristics of this atypical pathway to RI: upshear cloud migration and the TCA cloud patterns. As will be shown in the next section, it demonstrated a key feature in WV imagery, as well.

This evolutionary pattern appears in each of the other "atypical" RI TCs with only small variations among them. As examples, Fig. 14 contains the azimuthal variations of radially averaged brightness temperatures for Norbert, Fabio, Hilda, and Joaquin. 


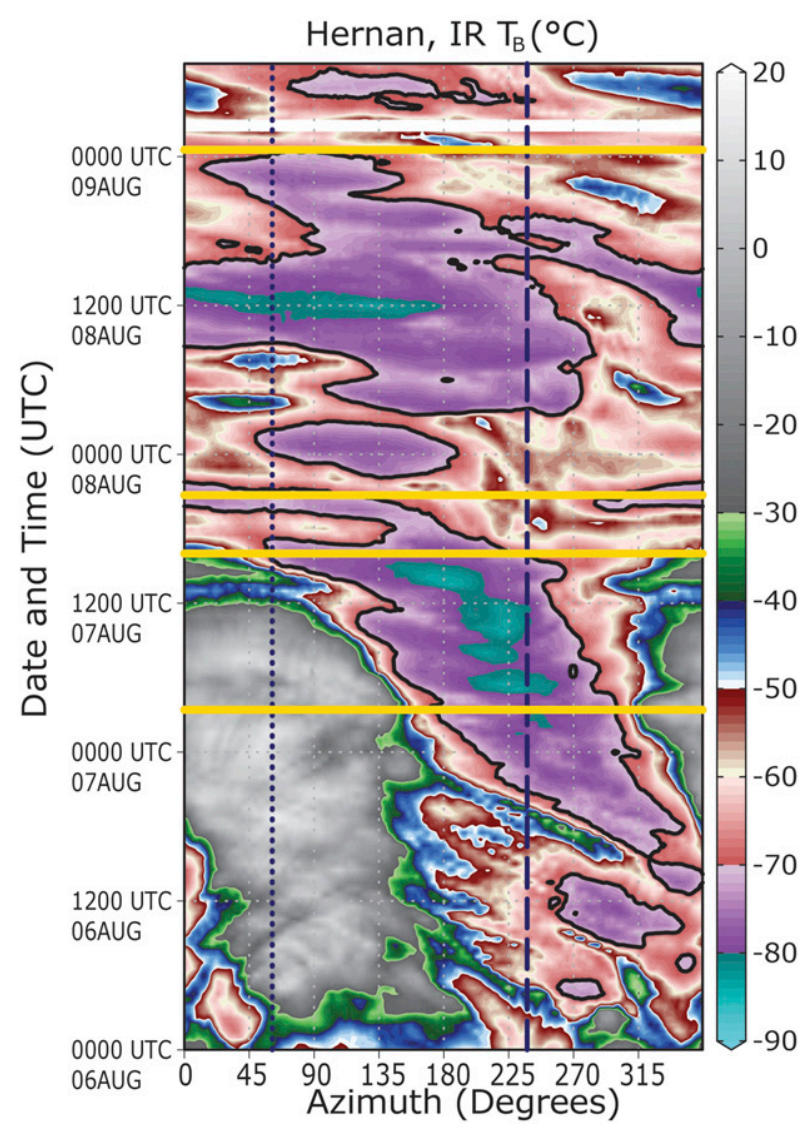

FIG. 12. Hovmöller diagram of brightness temperatures $\left({ }^{\circ} \mathrm{C}\right)$ averaged over the annulus of $80-130 \mathrm{~km}$ vs storm-relative azimuths. Angles are meteorological convention (clockwise/anticyclonic to the right), where $0^{\circ}$ is north, $90^{\circ}$ is east, and so on. Solid gold lines correspond to the snapshots in Fig. 11. The thick black line corresponds to the $-70^{\circ} \mathrm{C}$ contour. Dashed blue line is timeaveraged downshear angle; dotted blue line is time-averaged upshear angle.

All of them display this general progression of a long, slow upshear migration of clouds followed by repeating TCAs in the left-of-shear region. In Hilda (Fig. 14c), as a variant on this behavior, there are two separate instances of the upshear migration. In all cases, this migration requires at least $36 \mathrm{~h}$ to complete. What makes the convective activity unique after the upshear shift is that the coldest clouds are azimuthally bound-they do not completely wrap around the core of the TC. Contrast this behavior with examples of 2009 EPAC Rick and 2015 EPAC Patricia (Fig. 15). In these cases, directly before RI, the coldest cloud tops encircle the core in the span of a few hours. They do not exhibit the same azimuthally localized features as the atypical group. This behavior is coincident with discussions by Knaff (2008), where a large convective feature wraps around the core prior to RI.
To determine the periodicities of the convection in the six TCs, Fig. 16 shows the time series of all six "atypical" TCs and the total coverage of colder than $-70^{\circ} \mathrm{C}$ clouds. In addition, the right column of Fig. 16 displays the autocorrelation functions (further defined in the appendix) for each of the six TCs. Using Hernan as an example (Fig. 16b), the simplest way to understand the correlation function is by locating the largest anticorrelation lag (in Hernan's cases, this would be $2 \mathrm{~h} 15 \mathrm{~min}$ ). This measures the peak-trough lag. Doubling that number would then present a close approximation of the localized periodicity of the TCAs in terms of peak-to-peak measurement as analyzed by the wavelets (Fig. 13b). Generally, the derived periods using these methods are all between 4 and $8 \mathrm{~h}$. Table 3 summarizes the areal size and percentage changes associated with the TCAs. On average, they cover $5 \%$ the size of the total cloud field defined in Fig. 11: 10000$15000 \mathrm{~km}^{2}$.

Physically, these episodic, asymmetric convective maxima are associated with nutations of the tilt of the midlevel vortex superposed on a longer tilt realignment precession. A "nutation" can be defined as a higher-order rotation imposed on a longer, slower precession of a gyroscopic body. Nutations are unbalanced oscillations caused by overshooting deviations from a balanced precession (Fowles 1977, 243-245). An exploration of this phenomenon is presented in Part II.

\section{b. Water vapor imagery}

TCAs are not the only important characteristics of these "atypical" TCs. Two important markers of this behavior can also be noted in WV imagery: upshear warming/ drying and upshear arcs. The first feature of note is the near-permanent dichotomy in WV brightness temperature anomalies. Figure 17 shows the WV brightness temperature deviations from the azimuthal mean averaged over a $100-\mathrm{km}$ annulus from 250 to $350 \mathrm{~km}$ for two of the atypical TCs (Norbert and Joaquin) and two "classic" RI TCs (Rick and Emilia), which are only used for counterexamples (any of the four would suffice). All four time series are derived from genesis to the appearance of the IR eye. In the cases of Norbert and Joaquin, the coldest anomalies are consistently downshear and downshear left, while the warmest anomalies are upshear. This can be physically interpreted as cloud coverage focused downshear and downshear left, with clearer and warmer skies on the upshear side of the TCs. In the "classic" RI TCs (using Rick and Emilia as examples), this discernible pattern in the temperature anomalies is absent, with clouds potentially appearing on all sides. To quantify this behavior, we use a Fourier analysis, focusing on the azimuthal wavenumber- 1 asymmetry as a proxy for the upshear-downshear brightness temperature 

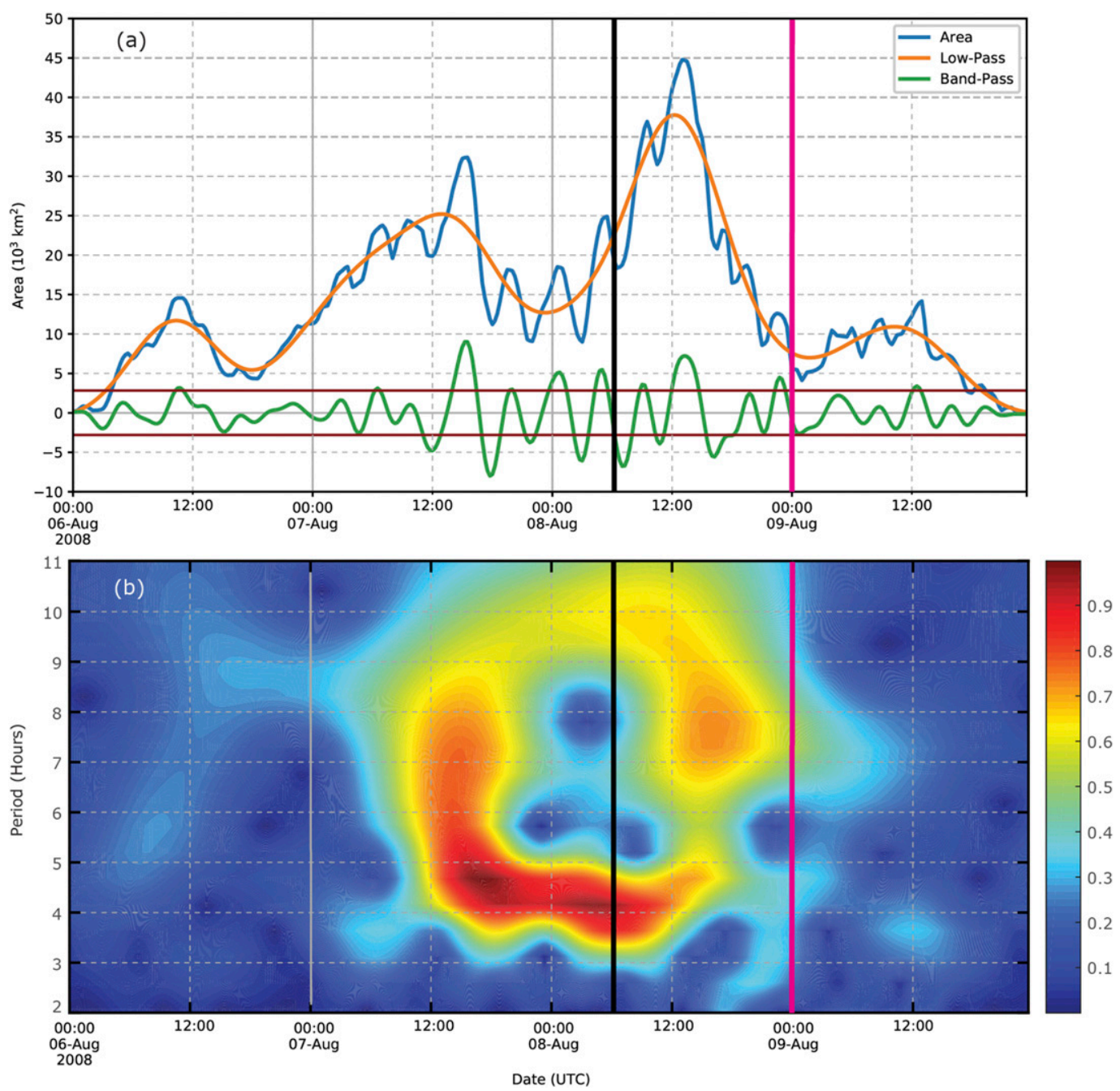

FIG. 13. (a) Areal coverage $\left(10^{3} \mathrm{~km}^{2}\right)$ of brightness temperatures colder than $-70^{\circ} \mathrm{C}$ within $200 \mathrm{~km}$ of Hernan's storm center (blue line), its low-pass-filtered state (orange line), and its bandpass-filtered state (green line). Dark red lines are \pm one standard deviation of the high-pass time series. Six TCAs appear starting at approximately 1400 UTC 7 Aug and cease appearing shortly after 1400 UTC 8 Aug. (b) Wavelet analysis of the high-pass time series [blue line minus orange line in (a)], where colored magnitude is ${ }^{\circ} \mathrm{C}$. In both, magenta line indicates appearance of eye, and black line indicates onset of RI according to HURDAT (see Fig. 1b).

dipole. Figure 18 illustrates the wavenumber-1 magnitude (Fig. 18a) and the phase (Fig. 18b) for the first $60 \mathrm{~h}$ of existence for Norbert, Joaquin, Emilia, and Rick. While the magnitudes are larger for Norbert and Joaquin, what is more indicative of the permanence of this asymmetry is the lack of variability in the phase of these asymmetries (Fig. 18b), especially when compared with Emilia and Rick.

The other significant feature is the presence of arcs-thin crescents of colder brightness temperatures along the leading edge of the cloud mass. These arcs appear exclusively upshear and are radially very thin. They are not the same feature discussed by Riemer et al. (2010), as their features are related to downdraft cooling, and ours are related to an upper-level evolution. Figure 19 is a collection of examples of these arcs in each of the "atypical" TCs, from the first appearance of this arc in Hernan (Fig. 19b), to subsequent appearances of the arcs in Norbert, Fabio, Hilda, and Joaquin (Figs. 19c-f), and finally to the edge of the cloud field upon the final stages of intensification of Guillermo (Fig. 19a). A more quantitative display is shown in Fig. 20. Radial cross sections are taken through each of the TCs at the times of Fig. 19. These snapshots indicate that for the three smaller TCs (Hernan, Fabio, and Hilda), the arcs exist approximately 


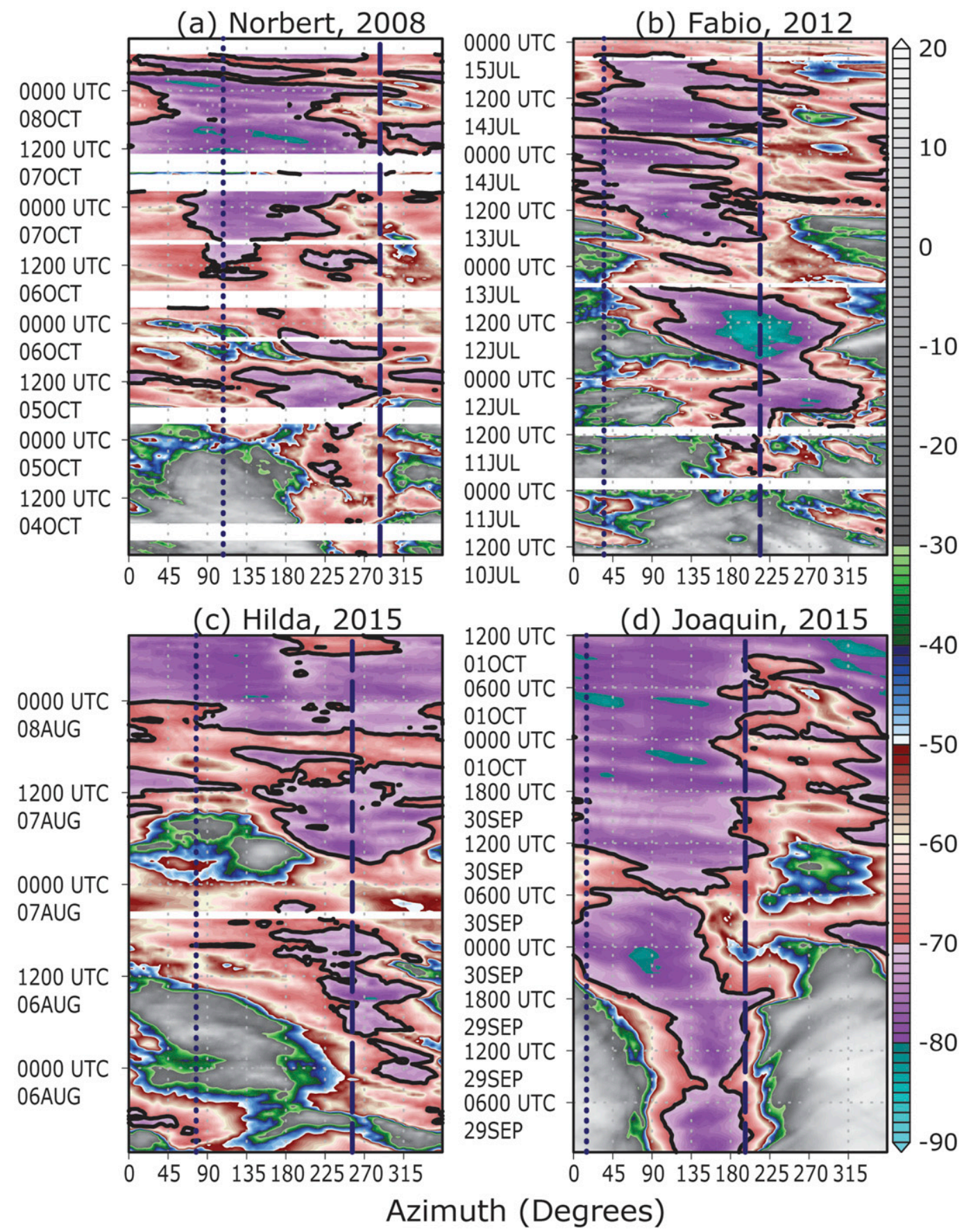

FIG. 14. As in Fig. 12, but for (a) Norbert, (b) Fabio, (c) Hilda, and (d) Joaquin.

$150 \mathrm{~km}$ from the center. For the three larger TCs (Guillermo, Norbert, and Joaquin), they are beyond $300 \mathrm{~km}$ from the center. In terms of brightness temperature, they exist in the range of $-40^{\circ}$ to $-60^{\circ} \mathrm{C}$. Radially, they are only $20-30 \mathrm{~km}$ wide. While a full quantitative, physical analysis is reserved for a future manuscript, we can state at this point that the WV imagery documents two important components of this atypical pathway to RI.

\section{c. Radial propagation speeds of cloud features}

Of the six "atypical" TCs, Joaquin provides a unique and clear opportunity to quantify the storm-relative radial propagation speeds of both the TCAs and the arcs 


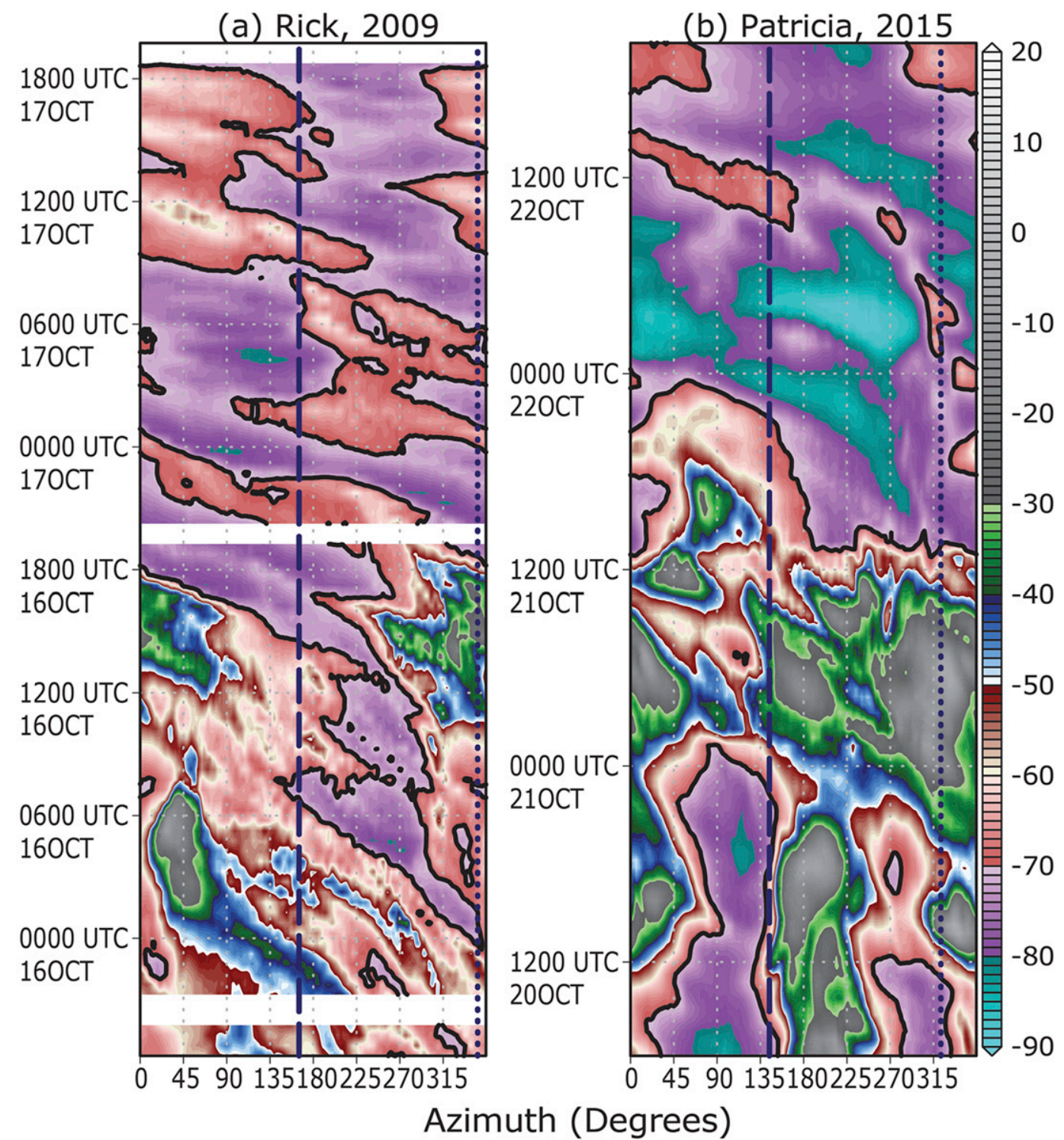

FIG. 15. As in Fig. 12, but for (a) Rick and (b) Patricia.

themselves. We apply both sequential IR and WV imagery in tandem, given the size of Joaquin's CDO, the temporal frequency ( $15 \mathrm{~min}$, as opposed to $30 \mathrm{~min}$ for the others) of the GOES-13 retrievals, and Joaquin's near-stationary movement at the time. In the $\mathrm{WV}$, the arc is fairly evident, as it is separated from the CDO by a warm gap (Fig. 19f). We define the edge of the TCA as the leading edge of the $-70^{\circ} \mathrm{C}$ contour. In Joaquin, this can be traced back to the eruption of a TCA near the core. Figure 21 is a time series of both IR and WV averaged over a small $5^{\circ}$ azimuthal slice due north of the TC. Tracking the warmer temperatures in the gap found in the IR image at earlier times indicates that the leading edge of the TCA initially has a radial propagation speed of approximately
$22.2 \mathrm{~m} \mathrm{~s}^{-1}$; however, the arc, as tracked in the WV imagery, has a propagation speed of approximately $6.4 \mathrm{~m} \mathrm{~s}^{-1}$, or roughly $70 \%$ slower than the initial outward movement of the clouds directly associated with the TCA in Joaquin.

Similar calculations were attempted for the other five "atypical" TCs. Using the same methodology as Fig. 20, the initial radial propagation speed of one of the TCAs from Hernan, for example, is calculated to be $16.4 \mathrm{~m} \mathrm{~s}^{-1}$. As another example, the radial propagation speed of the arc in Hilda (Fig. 19e) is $7.1 \mathrm{~m} \mathrm{~s}^{-1}$. Generally, the forward edges of the TCAs move with an initial radial speed of approximately $20 \mathrm{~m} \mathrm{~s}^{-1}$, while the arcs move with a radial speed of approximately $7 \mathrm{~m} \mathrm{~s}^{-1}$. 
(a) Guillermo
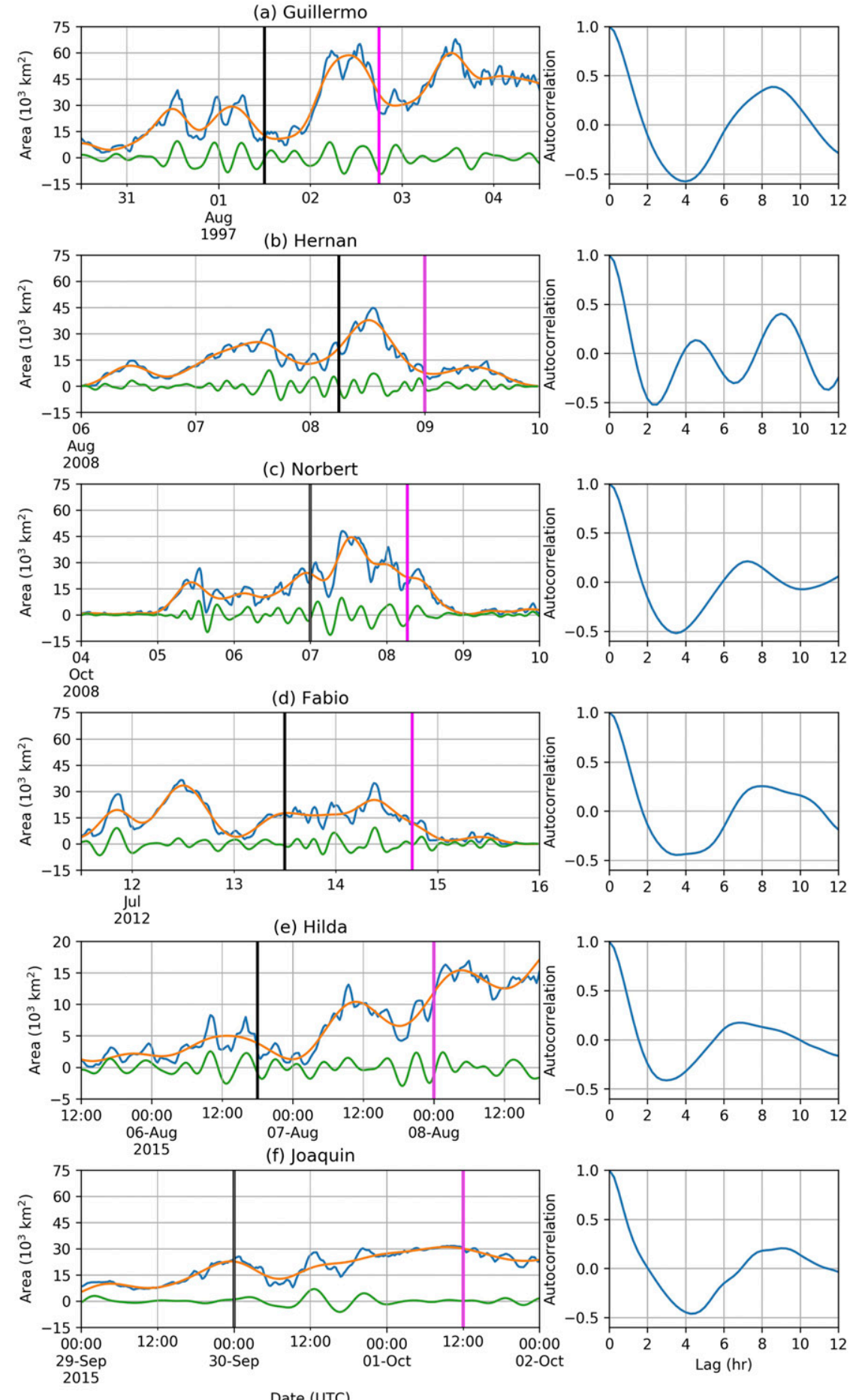

FIG. 16. (left) Areal coverage $\left(10^{3} \mathrm{~km}^{2}\right)$ of brightness temperatures colder than $-70^{\circ} \mathrm{C}$ within $200 \mathrm{~km}$ of the storm center (blue line), its low-pass-filtered state (orange line), and its bandpassfiltered state (green line) for (a) Guillermo, (b) Hernan, (c) Norbert, (d) Fabio, (e) Hilda, and (f) Joaquin. Black lines are onset of RI (see Fig. 1). Magenta line indicates appearance of the eye in IR imagery. (right) Respective autocorrelation functions for each TC's high-pass time series. 
TABLE 3. Variability of cloud coverage colder than $-70^{\circ} \mathrm{C}$ from TCAs for the six cases. Percent change is relative to storm size (see Fig. 7).

\begin{tabular}{lcc}
\hline \multicolumn{1}{c}{ Storm } & Areal change $\left(10^{3} \mathrm{~km}^{2}\right)$ & Percent change $(\%)$ \\
\hline 1997 Guillermo & 5 to 20 & 0.88 to 3.5 \\
2008 Hernan & 5 to 15 & 2.5 to 7.6 \\
2008 Norbert & 5 to 25 & 2.1 to 10.5 \\
2012 Fabio & 5 to 15 & 2.2 to 6.8 \\
2015 Hilda & 2 to 5 & 2.1 to 5.2 \\
2015 Joaquin & 15 & 4.8 \\
\hline
\end{tabular}

\section{Discussion}

Given that the focus of this manuscript is on observational evidence of a class of "atypical" RI TCs, the two outstanding questions here are as follows: 1) Fundamentally, how is a forecaster able to distinguish these TCs in an observational, impromptu sense? 2) Does this cloud structure merit its own identifying name?

The most important facts of which a forecaster must be aware are characteristics of the shear. While SHIPS shear may be an imperfect predictor of the environment around these TCs (Velden and Sears 2014), it can still be a useful metric if used as an appropriate guide, as shown in section 3. In our experience, the sequence of events follows this process:

1) Identify current storm intensity, explicitly looking for tropical storm strength.

2) Identify shear magnitude based on the SHIPS/ CIMSS analyses, explicitly looking for "moderate" $10-20 \mathrm{kt}\left(5-10 \mathrm{~m} \mathrm{~s}^{-1}\right)$.

3) If the shear magnitude falls within the range from the previous step, analyze $200-\mathrm{hPa}$ winds within a $20^{\circ}$ latitude and longitude circle to see if an upper-level anticyclone is responsible for the upper-level flow (see Fig. 7).

4) Using the shear direction, monitor IR imagery for pulsing, shape, and upshear expansion of TCAs and monitor WV imagery for upshear arcs in $-40^{\circ}$ to $-60^{\circ} \mathrm{C}$ range and for clearing/warming beyond the arcs.

In terms of general applicability, preliminary analysis indicates that this behavior is more frequent in the eastern Pacific than in the northern Atlantic due to a specific climatological setup. Figure 22 depicts the climatological winds, where "climatology" is defined as all Julys, Augusts, and Septembers from 1980 to 2010, over the two basins from ERA-Interim (Dee et al. 2011). The EPAC upper-level flow is dominated by an anticyclone. Assuming our theory holds, it stands to reason that this behavior would be favored in this basin, and there likely exist many more candidates here.

At this stage, these analyses are still subjective, and these cases were identified through subjective means. Furthermore, these are rare cases (sheared RI TCs) in a class of rare events (TCs that undergo RI). While a full climatological treatment is not a main goal of this study, we can briefly contextualize these findings using the 2004-14 TC seasons as a subsample. For simplicity, we focus on EPAC because of its potential favorability in a climatological sense. We then perform a simple first cut on the SHIPS database by extracting only those TCs that undergo RI $\left(15 \mathrm{~m} \mathrm{~s}^{-1}\right.$ increase in $24 \mathrm{~h}$ ) while either SHIPS shear calculation is greater than $5.4 \mathrm{~m} \mathrm{~s}^{-1}$ and either shear calculation has three consecutive entries $(12 \mathrm{~h})$ of shear greater than $5.4 \mathrm{~m} \mathrm{~s}^{-1}$ prior to the TCs becoming major hurricanes $\left(50 \mathrm{~m} \mathrm{~s}^{-1}\right)$. The latter criterion eliminates spurious shear variability in favor of sustained moderate shear. This methodology, when used on the entire dataset, will identify Guillermo, Hernan, Norbert, and Hilda, but it will not capture Fabio (fails both the RI requirement and the major hurricane requirement). Focusing on the 2004-14 time frame, this methodology does identify several more cases: 2004 Javier, 2004 Howard, 2006 Bud, 2006 Lane, 2009 Felicia, 2013 Raymond, 2014 Cristina, and 2014 Marie. Analyses of satellite imagery confirm the appearance of TCAs. Given this information, for the years 2004-14 in EPAC, there were a total of 203 depressions, of which 172 were named storms, of which 91 were hurricanes, and of which 42 were major hurricanes (Landsea and Franklin 2013). Of the 42 majors, 10 underwent "atypical" RI. While this sample is small, and while being cognizant of the fact that a proper climatological analysis is reserved for a future study (including planned automated analyses of satellite imagery), this simple SHIPS analysis indicates that a moderate fraction $(20 \%-25 \%)$ of the TCs that undergo RI to major hurricane status in EPAC follow the "atypical" RI path in sustained, moderate vertical wind shear.

We must stress that this cursory climatological inspection is not meant to imply that all TCs in this regime will undergo RI, as both Rios-Berrios and Torn (2017) and Finocchio and Majumdar (2017) demonstrated in a climatological way. Nor do all EPAC TCs undergo this process since the TC has to respond accordingly to the forcing, and this behavior is not exclusive to this basin. From a climatological perspective, it is simply more likely in EPAC because of the upper-level climatology. So while this behavior is rare and may be favored in a specific basin (EPAC), 


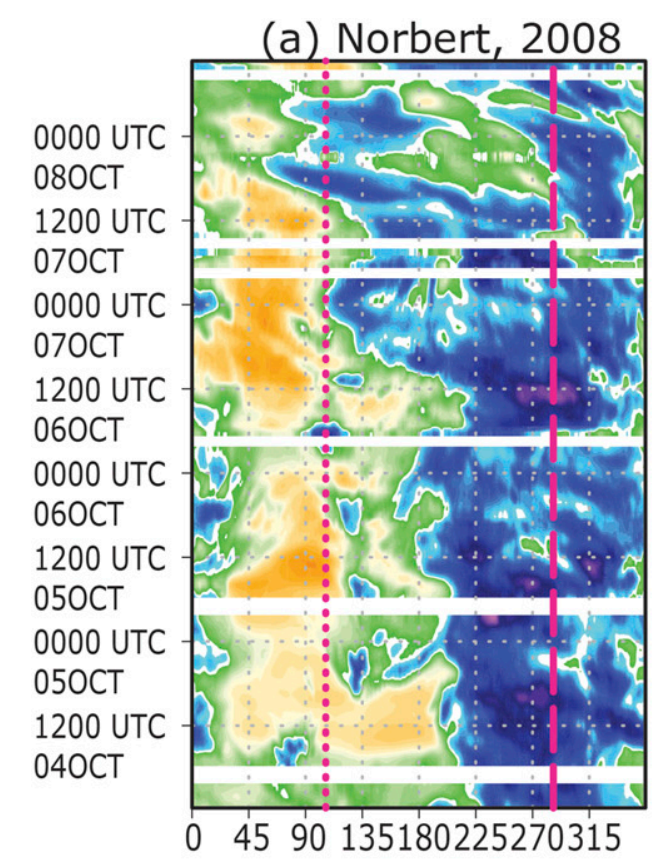

1200 UTC
$010 C T$
0600 UTC
$010 C T$
0000 UTC
$010 C T$
1800 UTC
30 SEP
1200 UTC
30 SEP
0600 UTC
30SEP
0000 UTC
30 SEP
1800 UTC
29SEP
1200 UTC
29SEP
0600 UTC
$29 S E P$

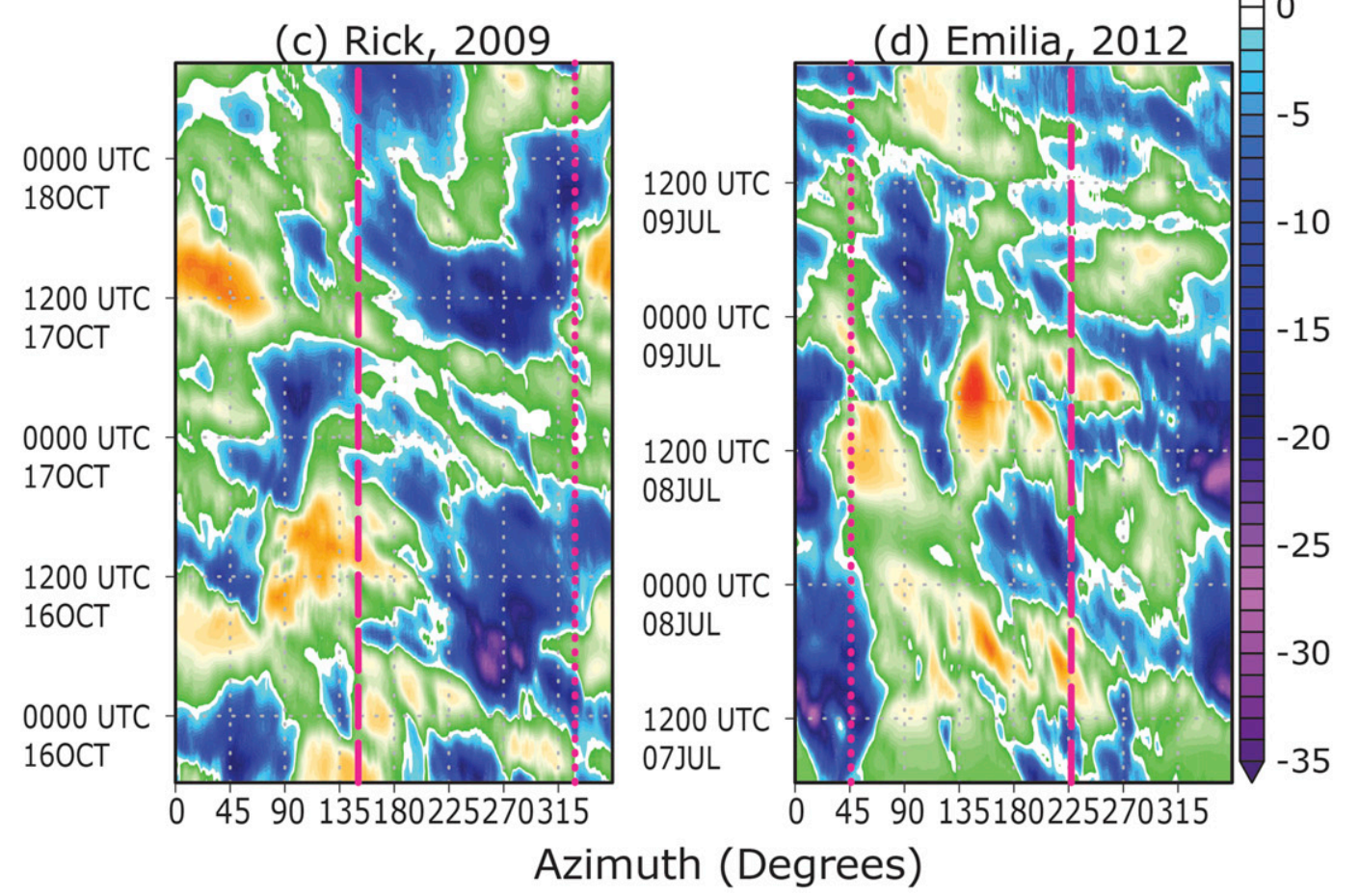

FIG. 17. Hovmöllers of WV brightness temperature anomalies $\left({ }^{\circ} \mathrm{C}\right)$ in the annulus from 250 to $350 \mathrm{~km}$ vs azimuth, where anomalies are departures from the azimuthal mean, for (a) Norbert, (b) Joaquin, (c) Rick, and (d) Emilia. Dashed magenta lines are time-averaged downshear; dotted magenta lines are time-averaged upshear. Angles are meteorological convention. All are smoothed with a three-point running mean.

Joaquin demonstrates that this process can occur anywhere, although this evolution will be more dependent on day-to-day synoptic variability in other basins. In addition, there are other cases that do not necessarily undergo RI or reach major hurricane status but still exhibit TCAs and proceed to intensify, albeit not rapidly, in moderate shear (such as Fabio). More research is needed to reduce the degrees of freedom in the relationship between TCAs and intensification rates in moderate shear. 

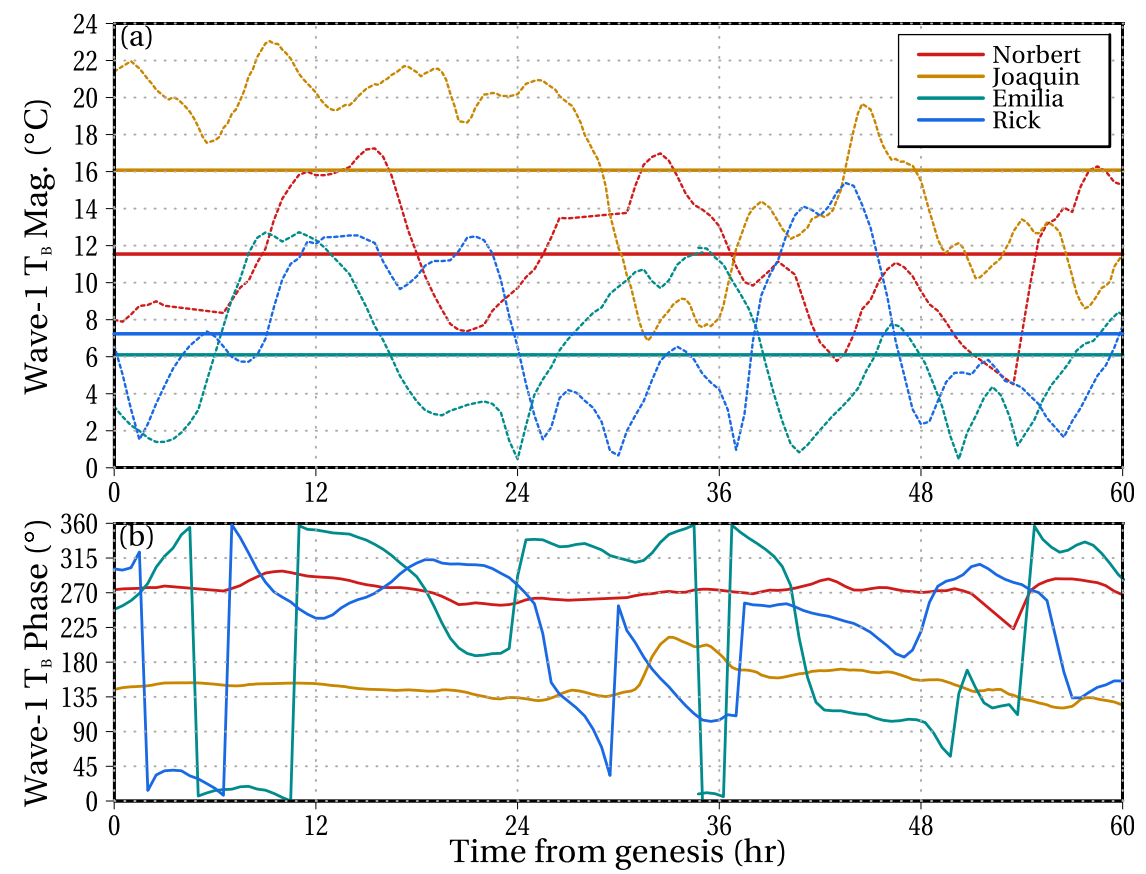

FIG. 18. (a) Wavenumber-1 Fourier magnitude and (b) phase of averaged WV brightness temperatures (see Fig. 17) for the first $60 \mathrm{~h}$ of existence for Norbert, Joaquin, Emilia, and Rick. In (a), dashed lines are the time series, while solid thick lines are the averages over the whole time series.

As far as giving this its own name, we feel that the distinct cause of these structures requires that they be named (see Part II). Convective bursts of various shapes and sizes have been documented previously, and sheared TCs have been studied extensively. Zawislak et al. (2016) and Rogers et al. (2016) documented convective bursts that migrated into the upshear-left quadrant in observations of 2014 NATL Edouard, and they argued that played a role in Edouard's gradual intensification to a major hurricane. Molinari et al. (2013) discussed bursts and downdraft cooling in 2002 NATL Edouard. Nguyen et al. (2017) discussed symmetric convection differences between two sheared tropical storms. While they all briefly analyzed IR satellite imagery, they did not consider a periodic, temporal component of their bursts, which is a significant finding of the work performed here. Reasor et al. (2009), in their analysis of Guillermo, did put periods to different motions in their work (e.g., $140 \mathrm{~min}$ for an elliptical eyewall), but they did not directly relate the tilt to convective periodicity. We demonstrate in Part II that this periodicity can be explained by tilt nutations. We have also provided a temporal periodicity of these events: $4-8 \mathrm{~h}$.

In terms of separating this feature from a mesoscale convective system (MCS) identified by Rappin and Nolan (2012) or Harr and Elsberry (1996), we would admit that calling these features MCSs is technically not incorrect, as the definition of "MCS" appears to be quite encompassing (e.g., Sanders and Emanuel 1977; Hodges and Thorncroft 1997; Elsner and Kara 1999; Carbone et al. 2002); however, this downplays the role that the tilt plays in modulating these convective structures. Rappin and Nolan (2012) focused, as Zawislak et al. (2016) and Rogers et al. (2016) did, on the upshear migration of convection but only loosely associated it with the tilt of the vortex. Rappin and Nolan (2012) never discussed higher-frequency nutations. Additionally, climatological studies that have used composites (Corbosiero and Molinari 2002; Chen et al. 2006; DeHart et al. 2014; Reasor et al. 2013) have found that convection is usually downshear left, but this type of analysis inherently masks potentially important smallerscale details. For this specific class of TCs discussed in this manuscript, the tilt and its higher-frequency nutations are central components of this evolution; therefore, we feel that given the specific cause of the modulation (the vortex tilt nutation; see Part II) and its repetitive, periodic, potentially predictable nature, it deserves its own classification: a tilt-modulated convective asymmetry.

\section{Summary}

Six tropical cyclones (TCs) that unexpectedly underwent rapid intensification (RI) in moderate vertical 
(a) Guillermo, 1600 UTC 04 Aug

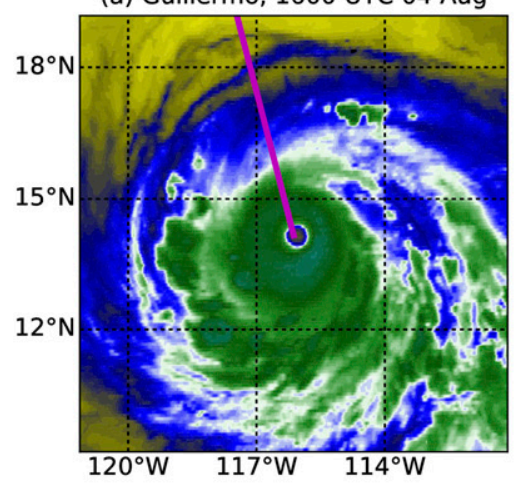

(c) Norbert, 1715 UTC 07 Oct

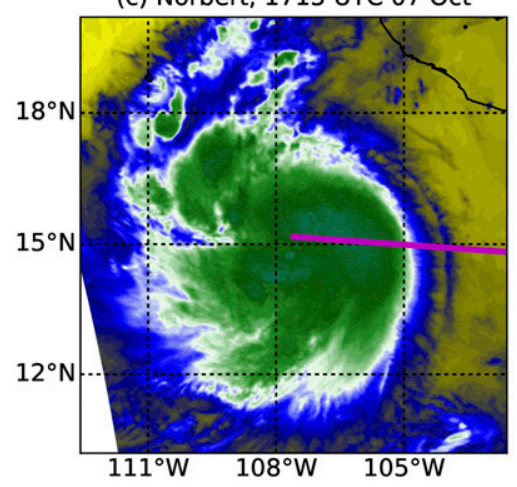

(e) Hilda, 1300 UTC 07 Aug

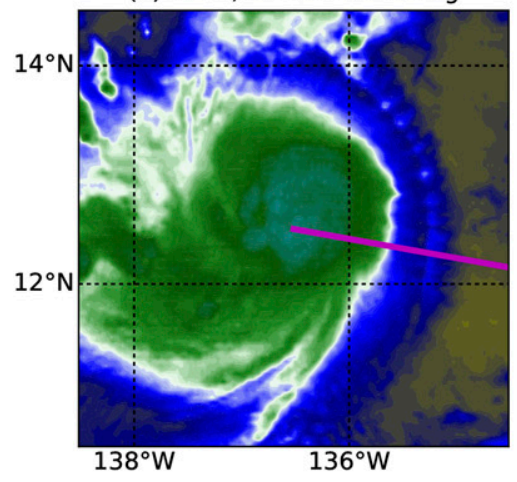

(b) Hernan, 1500 UTC 07 Aug

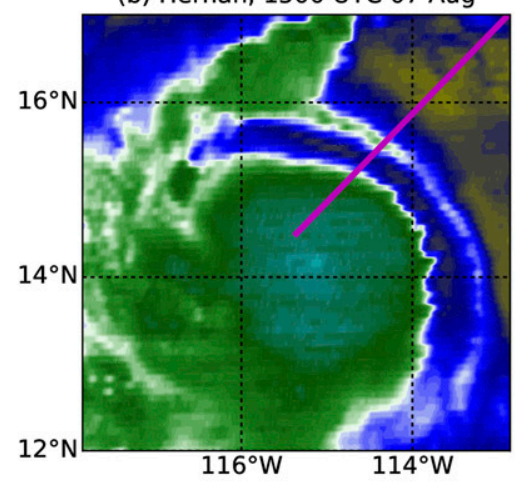

(d) Fabio, 0600 UTC 14 Jul
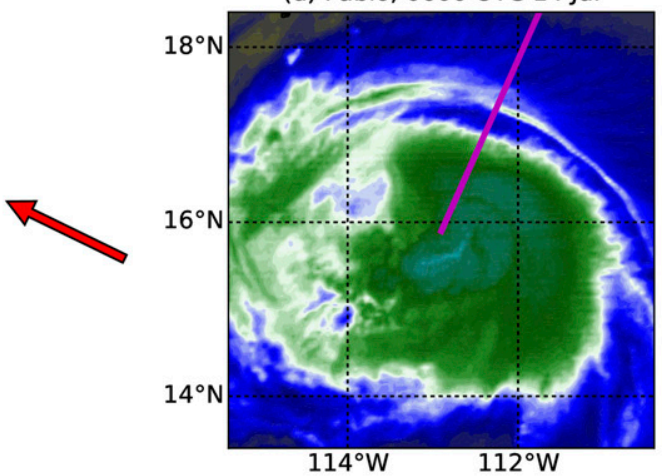

(f) Joaquin, 0315 UTC 01 Oct

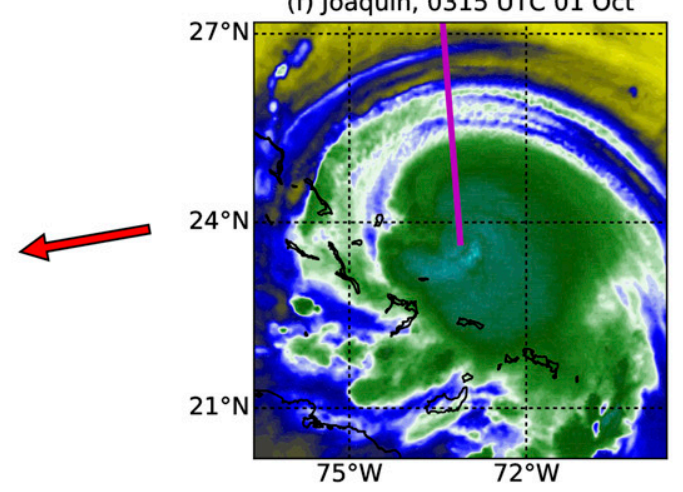

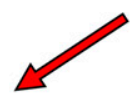
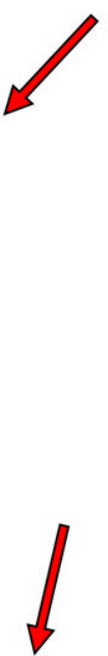

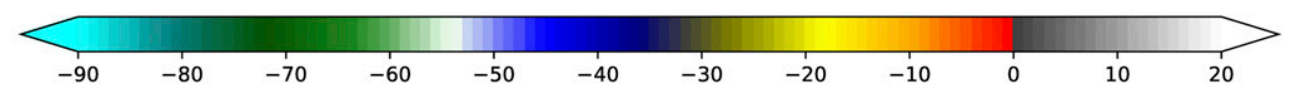

FIG. 19. WV satellite imagery of upshear arcs in the six TCs: (a) Guillermo, (b) Hernan, (c) Norbert, (d) Fabio, (e) Hilda, and (f) Joaquin. Guillermo's snapshot [(a)] highlights the end of this process, while the other five capture these arcs in the middle of each storm's respective intensifications. Red arrows indicate shear vectors. Magenta lines indicate the radial profiles of Fig. 20.

wind shear (i.e., between 5 and $10 \mathrm{~m} \mathrm{~s}^{-1}$ ) are investigated using both infrared (IR) and water vapor (WV) geostationary satellite imagery, the Statistical Hurricane Intensity Prediction System (SHIPS), and reanalysis data. The six TCs are 1997 eastern Pacific (EPAC) Guillermo, 2008 EPAC Hernan, 2008 EPAC Norbert, 2012 EPAC Fabio, 2015 EPAC Hilda, and 2015 northern
Atlantic (NATL) Joaquin. In terms of SHIPS analyses, all of the TCs underwent RI in conditions where the shear is one to five standard deviations higher than the climatological average of storms undergoing RI. The average $200-850$-hPa shear value over all six TCs is $7.5 \mathrm{~m} \mathrm{~s}^{-1}$. In terms of satellite analyses, Hernan is specifically used as a prototype to illustrate the different 


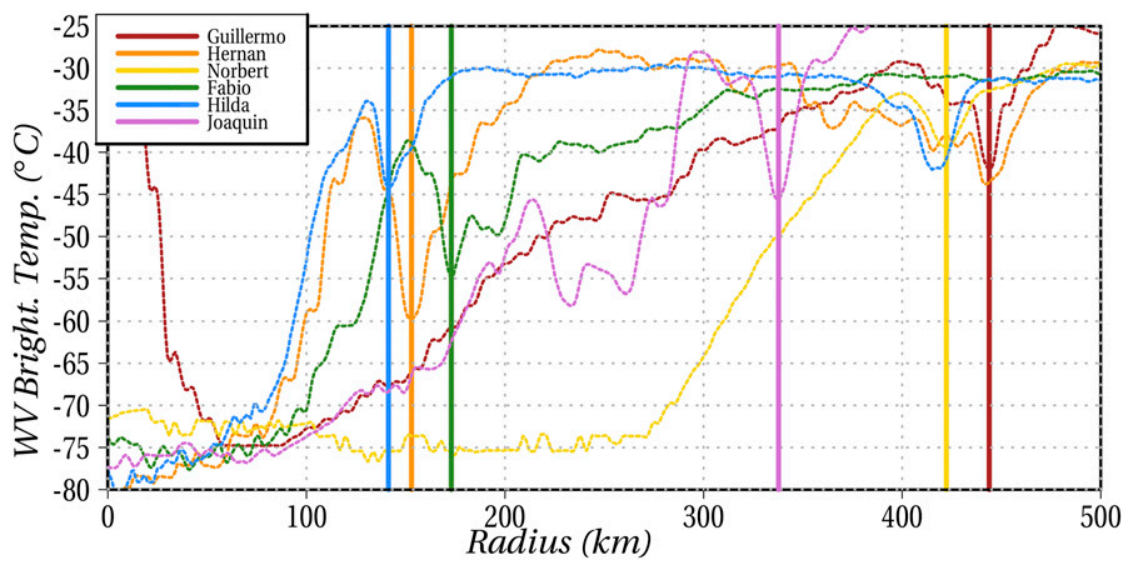

FIG. 20. Radial profiles of WV brightness temperature $\left({ }^{\circ} \mathrm{C}\right)$ from Fig. 19. Solid vertical lines indicate the arcs.

stages of these TCs during intensification. The two unique characteristics in IR observations are a slowly precessing upshear shift in the cloud shield and the appearance of repeating convective structures, herein defined as a tiltmodulated convective asymmetry (TCA). The upshear shift takes 1-2 days to complete. Following the shift, the TCAs pulse. These TCAs all have a similar distinct shape, a temperature boundary $\left(-70^{\circ} \mathrm{C}\right)$, and a general upshear expansion relative to the shear vector. Using a windowed-sinc filter on a time series of total area coverage of brightness temperatures colder than $-70^{\circ} \mathrm{C}$ in the IR band, a series of six TCAs can be identified in Hernan. Using a wavelet analysis for quantification, the TCAs in Hernan pulse with a periodicity of approximately $4.5 \mathrm{~h}$. Applying similar filtering to the additional five TCs provides a range of this periodicity as calculated by wavelets and the autocorrelation function: 4-8h. In sum, a TCA can be defined thusly: an azimuthally bound, upshear-expanding cloud feature encapsulated by the $-70^{\circ} \mathrm{C}$ IR isotherm that pulses with a periodicity of $4-8 \mathrm{~h}$, expands at a radial rate of between 15 and $25 \mathrm{~m} \mathrm{~s}^{-1}$, is generally responsible for a $5000-20000 \mathrm{~km}^{2}$ change in cloud coverage colder than $-70^{\circ} \mathrm{C}$, and whose coldest cloud-top temperatures are below $-80^{\circ} \mathrm{C}$. Part II will add further dynamic and thermodynamic constraints.

In addition to the IR analyses, WV analyses also indicate a few interesting characteristics. Using Joaquin and Norbert as examples, a localized area of warming/ drying exists upshear of the TCs. In addition, as was shown in all six, radially thin arcs exist exclusively upshear. These arcs range in $\mathrm{WV}$ brightness temperatures between $-40^{\circ}$ and $-60^{\circ} \mathrm{C}$ and are only on the order of $\sim 30 \mathrm{~km}$ wide. A warm gap separates all of these arcs from the main cloud shield. For the three smaller TCs (Hernan, Fabio, and Hilda), these arcs existed approximately $150 \mathrm{~km}$ from the core. For the three larger TCs (Guillermo, Norbert, and Joaquin), these arcs were farther than $300 \mathrm{~km}$ from the core. In general, the cloud exhaust of the TCAs expands with a radial speed of approximately $20 \mathrm{~m} \mathrm{~s}^{-1}$, while the arcs propagate with a speed of approximately $7 \mathrm{~m} \mathrm{~s}^{-1}$.
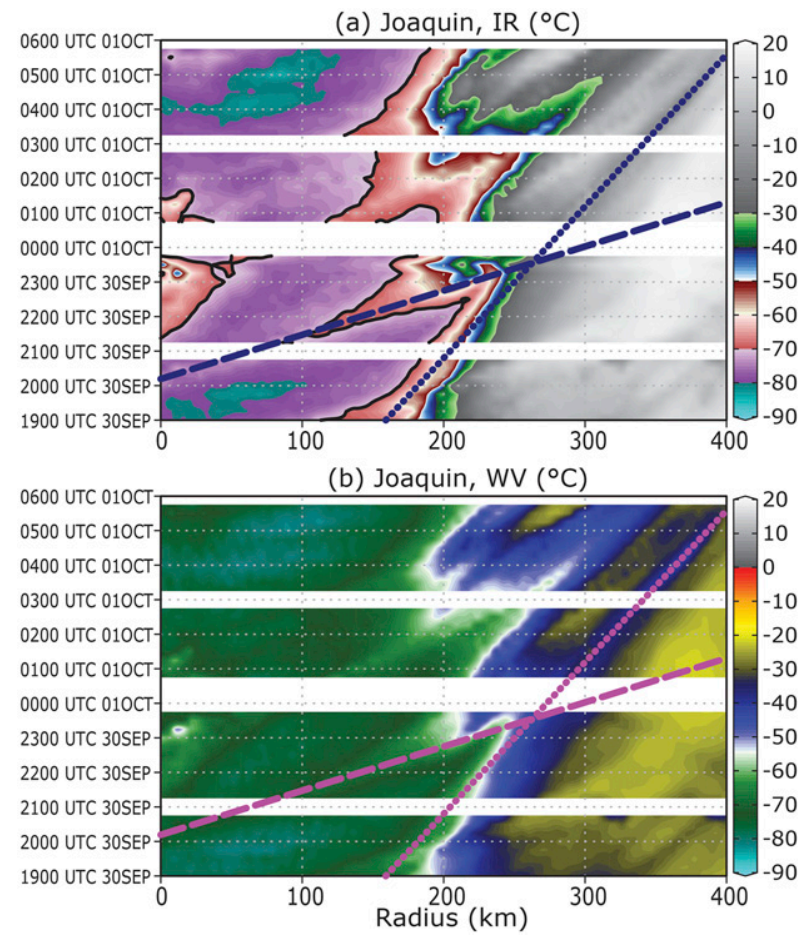

FIG. 21. Hovmöller diagrams of (a) IR and (b) WV brightness temperatures $\left({ }^{\circ} \mathrm{C}\right)$ averaged over a small $5^{\circ}$ azimuthal wedge due north of 2015 NATL Joaquin. The dashed line is the initial propagation speed of the cloud field associated with the TCA: $22.2 \mathrm{~m} \mathrm{~s}^{-1}$. The dotted line is the propagation speed associated with one of the arcs: $6.38 \mathrm{~m} \mathrm{~s}^{-1}$. 

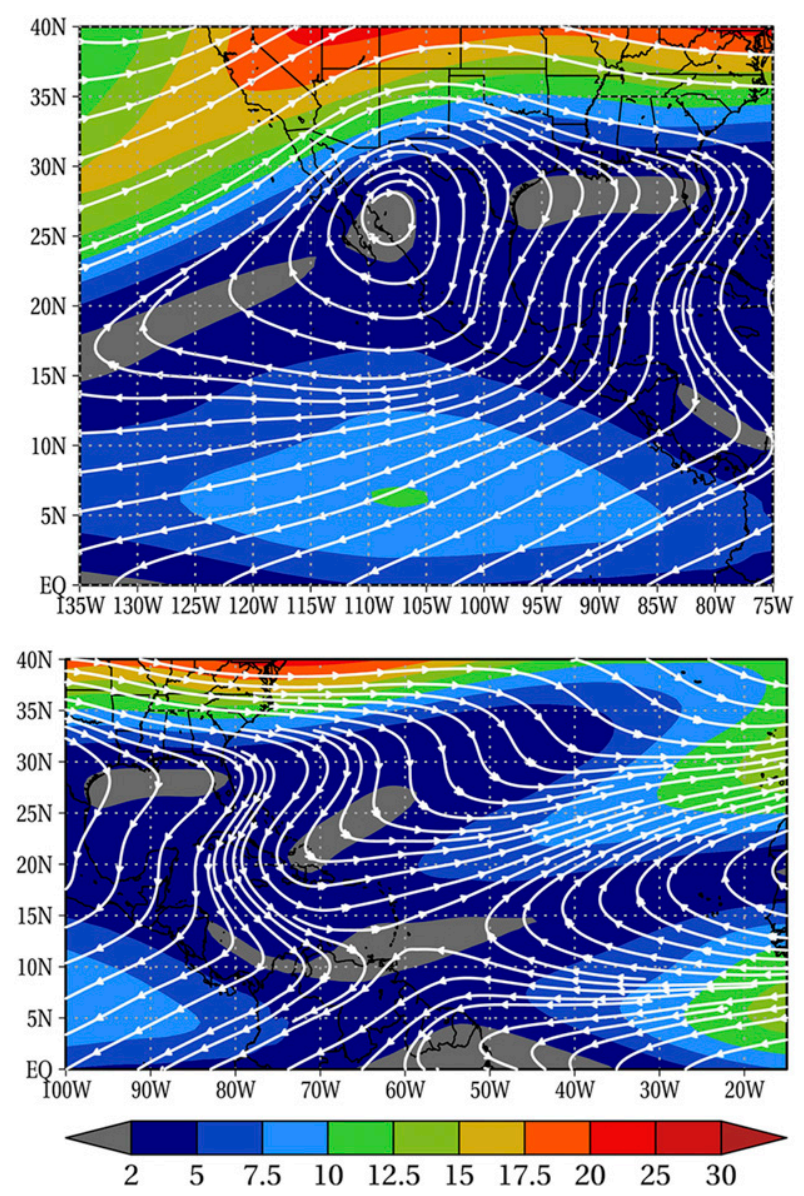

FIG. 22. Climatological (1980-2010) July-September 200-hPa winds $\left(\mathrm{m} \mathrm{s}^{-1}\right)$ from ERA-Interim over (top) EPAC and (bottom) NATL basins.

From a synoptic perspective, one of the most important characteristics is the structure of the vertical wind shear. For all six TCs, the upper-level environmental flow near the TC is controlled by a proximate upper-level anticyclone. Using Joaquin as an example-as Joaquin was the only one of the six to endure high shear (at least three standard deviations above climatology) by both an upper-level anticyclone and an upper-level trough in rapid succession-reanalysis indicates that winds from anticyclones are limited in the vertical to near the tropopause, a fact that some previous studies have indicated is more favorable for intensification (Elsberry and Jeffries 1996; Finocchio et al. 2016). This also matches theoretical studies of anticyclonic tropopause PV anomalies (Hoskins et al. 1985; Wirth 2001). We believe that given the analysis here, it may be worthwhile to investigate other TCs that intensified in shear, such as 2010 NATL Earl (Stevenson et al. 2014; Rogers et al. 2015) or 2014 NATL Edouard (Zawislak et al. 2016; Rogers et al. 2016).

The current identification process of TCs of this class is very subjective in nature. In terms of using an objective method to determine the presence of this class of TC in a climatological sense, we demonstrated that performing a filtered analysis on the periodicity of the IR cloud field is necessary but not exclusively sufficient to characterize this class of TCs. A further scene analysis must be performed to capture both the correct shape and the correct position of the cloud structures, along with additional features in WV imagery. Scene analyses through machine learning may be a promising way forward to identify TCs of this class. We believe we have identified clear markers indicating the presence of this class of TCs and have provided sufficient identifying phenomena to act as benchmarks to guide future modeling studies. In Part II, we focus entirely on the TCAs themselves in a modeling study, including the key dynamics influencing them and their spatiotemporal characteristics. As a small, final note, we will address the importance of the WV imagery in a future manuscript, as it illustrates a key process in this atypical RI evolution.

Acknowledgments. DRR graciously acknowledges a National Research Council Postdoctoral Research Award at the Naval Research Laboratory in Monterey, California. This research was supported by the Office of Naval Research PE 0601153N, as part of the Tropical Cyclone Intensity Departmental Research Initiative (TCI-DRI). We would like to acknowledge Chris Velden, Derrick Herndon, and David Stettner at the Cooperative Institute for Meteorological Satellite Studies for assistance and helpful discussions. Imagery in this paper has been produced using python's Matplotlib and Basemap toolkits, Matlab, gnuplot, and the Grid Analysis and Display System (GrADS). OISST data are kindly provided by the NOAA/OAR/ ESRL PSD in Boulder, Colorado, from their website (http:// www.esrl.noaa.gov/psd/). In addition, DRR would like to thank the following people for discussion of this topic over the past few years: Robert Hart, Paul Reasor, Kevin Viner, Yi Jin, Raymond Lee, Jerome Schmidt, James Campbell, Buck Sampson, and Jason Nachamkin. DRR would also like to acknowledge Nancy Merckle and the rest of the team at NESDIS and SSD web services for aid in satellite image recovery. We also thank John Knaff, Chris Velden, and one anonymous reviewer for their helpful comments, which greatly improved this work's clarity and content.

\section{APPENDIX}

\section{Signal Processing Methodology}

In section 4 , time series of areal coverages of the storm-centered cloud shield are presented. To convey the variability and time scales of the coverage changes 
properly, different signal processing tools are used. The first is a filter that is used in both low-pass and bandpass formulations. The filter used is a windowed-sinc filter (Smith 2003, 285-300):

$$
\begin{aligned}
F(t) & =f(t) \times \operatorname{sinc}\left[2 f_{c}\left(t-\frac{N-1}{2}\right)\right]\left[a_{0}-a_{1} \cos \left(\frac{2 \pi t}{N-1}\right)\right. \\
& \left.+a_{2} \cos \left(\frac{4 \pi t}{N-1}\right)-a_{3} \cos \left(\frac{6 \pi t}{N-1}\right)\right] .
\end{aligned}
$$

The window function [which contains the cosines in (A1)] used is the Blackman-Nuttall window, a window that is designed to minimize sidelobe response, otherwise known as Gibbs ringing or spectral leakage (Nuttall 1981). The data are first resampled to 15-min frequency to generalize the process for TCs that cross the GOES-East/West terminator. The missing data are filled in using piecewise cubic splines. The cutoff frequency $f_{c}$ is $0.02-$ a period of $12.5 \mathrm{~h}$-in order to filter everything occurring more frequently than any potential diurnal signal. The width of the window $N$ is 201 data points, which corresponds to a frequency rolloff of 0.02 ; edges are zero padded. The key reason for the initial filtering is that the diurnal signal is the dominant response in this cloud field and thus partially masks the signal of the TCAs.

The sequence of events for analysis is compute raw time series, determine low-pass signal, and then subtract the low-pass signal from the full signal. This resultant time series is the "high pass" time series. The wavelet and the autocorrelation are then performed on this time series. The filter in Eq. (A1) is used again to create a bandpass filter using spectral inversion, isolating the behavior between $12.5 \mathrm{~h}$ and a lower bound. The lower bound is prescribed to eliminate any higher-order convective activity (i.e., Reasor et al. 2009) and to clean the signal for visualization and is informed by the following wavelet analysis.

For determining the periodicity and temporal variability of convective activity near the storm center, a wavelet transform is used. Here, the Morlet wavelet, which is a Gaussian (window) convolved with a plane wave (transform), is used. In general form (Torrence and Compo 1998), the Morlet wavelet is

$$
\Psi_{0}(t)=\pi^{-1 / 4} e^{i \omega_{0} t} e^{-t^{2} / 2},
$$

which, much like the windowed-sinc filter, contains a frequency component [in this case, it is a plane wave $\left.\left(e^{i \omega_{0} t}\right)\right]$ convolved with a time component [in this case, a Gaussian function $\left(e^{-t^{2} / 2}\right)$ ]. The justification for using a wavelet is that wavelets were originally developed for analyzing small packets of activity embedded within a larger, nonperiodic time series. Torrence and Compo (1998), for example, used the wavelet to extract multiyear signals embedded in a time series of El Niño. Given the results of the wavelet, the lower bound for the bandpass filter is half the derived period from the wavelets.

The simplest way to think of a wavelet is as a combination of a frequency analysis (similar to a Fourier transform) and a temporal analysis (similar to a running mean). This allows for it to detect oscillations that are localized in time. The way to interpret wavelet results (Fig. 13) is by considering each time period on the ordinate $(1,2,3 \mathrm{~h}$, etc.) as its own power spectrum. The wavelet indicates that at a given point in a time series, the time series has an oscillation whose signal is strongest at a given period. In Hernan's case, the strongest signal is $4.25 \mathrm{~h}$ for the $24 \mathrm{~h}$ before the eye appears. Note how this signal disappears after the eye forms. If one were to perform a Fourier analysis on this time series, it would document that the time series has a dominant frequency, but it would be unable to indicate where in the time series that frequency is localized. The wavelet permits this analysis, which is critical for our analysis to indicate where the TCAs are occurring.

Since the continuous wavelet analysis results in a complicated visualization, a related, simplified visualization can be achieved with an autocorrelation function (Wilks 2011, 57-60), where an autocorrelation function measures the correlation of a time series lagged with itself. The autocorrelation is calculated using the Wiener-Khinchin theorem, which uses the more familiar Fourier transforms. It must be stressed that the autocorrelation is only shown as a visual simplification of the wavelet, since the wavelet operates in both the time domain and the frequency domain, while an autocorrelation function (and the Fourier transform) only operates in the frequency domain (Smith 2003). As noted, since one of the phenomena we discuss occurs at a given frequency but is localized in time, the wavelet can identify both the time frame and the frequency, while frequency-only analyses cannot. An example is shown in section 4 that demonstrates how the two calculations yield complementing results.

\section{REFERENCES}

Avila, L., 2008: Tropical Storm Hernan discussion 7. NOAA/NHC Rep. EP092008, https:/www.nhc.noaa.gov/archive/2008/ep09/ ep092008.discus.007.shtml.

Berg, R., 2012: Hurricane Fabio discussion 7. NOAA/NHC Rep. EP062012, http://www.nhc.noaa.gov/archive/2012/ep06/ep062012. discus.007.shtml.

Beven, J. L., 2015a: Hurricane Hilda discussion 8. NOAA/NHC Rep. EP102015, http:/www.nhc.noaa.gov/archive/2015/ep10/ ep102015.discus.008.shtml.

, 2015b: Hurricane Joaquin discussion 12. NOAA/NHC Rep. AL112015, http://www.nhc.noaa.gov/archive/2015/al11/al112015. discus.013.shtml. 
Black, P. G., and R. A. Anthes, 1971: On the asymmetric structure of the tropical cyclone outflow layer. J. Atmos. Sci., 28, 1348-1366, https://doi.org/10.1175/1520-0469(1971)028<1348: OTASOT $>2.0 . \mathrm{CO} ; 2$.

Blake, E., 2009: Tropical Storm Rick discussion 2. NOAA/NHC Rep. EP202009, http://www.nhc.noaa.gov/archive/2009/ep20/ ep202009.discus.002.shtml.

- 2012: Tropical Storm Emilia discussion 5. NOAA/NHC Rep. EP052012, http://www.nhc.noaa.gov/archive/2012/ ep05/ep052012.discus.005.shtml.

Brown, D., 2008: Hurricane Norbert discussion 14. NOAA/NHC Rep. EP152008, http://www.nhc.noaa.gov/archive/2008/ep15/ ep152008.discus.014.shtml.

_- 2015: Hurricane Joaquin discussion 13. NOAA/NHC Rep. AL112015, http://www.nhc.noaa.gov/archive/2015/al11/ al112015.discus.013.shtml.

Carbone, R. E., J. D. Tuttle, D. A. Ahijevych, and S. B. Trier, 2002: Inferences of predictability associated with warm season precipitation episodes. J. Atmos. Sci., 59, 2033-2056, https://doi.org/ 10.1175/1520-0469(2002)059<2033:IOPAWW>2.0.CO;2.

Chen, S. S., J. A. Knaff, and F. D. Marks, 2006: Effects of vertical wind shear and storm motion on tropical cyclone rainfall asymmetries deduced from TRMM. Mon. Wea. Rev., 134, 3190-3208, https://doi.org/10.1175/MWR3245.1.

Corbosiero, K. L., and J. Molinari, 2002: The effects of vertical wind shear on the distribution of convection in tropical cyclones. Mon. Wea. Rev., 130, 2110-2123, https://doi.org/ 10.1175/1520-0493(2002)130<2110:TEOVWS >2.0.CO;2.

Dee, D. P., and Coauthors, 2011: The ERA-Interim reanalysis: Configuration and performance of the data assimilation system. Quart. J. Roy. Meteor. Soc., 137, 553-597, https://doi.org/ 10.1002/qj.828.

DeHart, J. C., R. A. Houze, and R. F. Rogers, 2014: Quadrant distribution of tropical cyclone inner-core kinematics in relation to environmental shear. J. Atmos. Sci., 71, 2713-2732, https://doi.org/10.1175/JAS-D-13-0298.1.

Delaunay, B., 1934: Sur la sphère vide. Bull. l'Académie Sci. l'URSS, Classe Sci. Math. Nat., 6, 793-800.

DeMaria, M., M. Mainelli, L. K. Shay, J. A. Knaff, and J. Kaplan, 2005: Further improvements to the Statistical Hurricane Intensity Prediction Scheme (SHIPS). Wea. Forecasting, 20, 531-543, https://doi.org/10.1175/WAF862.1.

Didlake, A. C., and R. A. Houze, 2013: Convective-scale variations in the inner-core rainbands of a tropical cyclone. J. Atmos. Sci., 70, 504-523, https://doi.org/10.1175/JAS-D-12-0134.1.

Dunion, J. P., C. D. Thorncroft, and C. S. Velden, 2014: The tropical cyclone diurnal cycle of mature hurricanes. Mon. Wea. Rev., 142, 3900-3919, https://doi.org/10.1175/MWR-D-13-00191.1.

Dvorak, V. F., 1975: Tropical cyclone intensity analysis and forecasting from satellite imagery. Mon. Wea. Rev., 103, 420-430, https:// doi.org/10.1175/1520-0493(1975)103<0420:TCIAAF > 2.0.CO;2.

_ 1984: Tropical cyclone intensity analysis using satellite data. NOAA Tech. Rep. NESDIS 11, 45 pp.

Elsberry, R. L., and R. A. Jeffries, 1996: Vertical wind shear influences on tropical cyclone formation and intensification during TCM-92 and TCM-93. Mon. Wea. Rev., 124, 1374-1387, https://doi.org/ 10.1175/1520-0493(1996)124<1374:VWSIOT>2.0.CO;2.

Elsner, J. B., and A. B. Kara, 1999: Hurricanes of the North Atlantic: Climate and Society. Oxford University Press, 488 pp.

Finocchio, P. M., and S. J. Majumdar, 2017: A statistical perspective on wind profiles and vertical wind shear in tropical cyclone environments of the Northern Hemisphere. Mon. Wea. Rev., 145, 361-378, https://doi.org/10.1175/MWR-D-16-0221.1.
- - - D. S. Nolan, and M. Iskandarani, 2016: Idealized tropical cyclone responses to the height and depth of environmental wind shear. Mon. Wea. Rev., 144, 2155-2175, https://doi.org/10.1175/MWR-D-15-0320.1.

Fowles, G. R., 1977: Analytical Mechanics. 3rd ed. Holt, Rinehart, and Winston, $334 \mathrm{pp}$.

Franklin, J., 2005: Hurricane Wilma discussion 12. NOAA/NHC Rep. AL242005, http://www.nhc.noaa.gov/archive/2005/dis/ al242005.discus.012.shtml.

Gallina, G. M., 2002: Environmental vertical wind shear and tropical cyclone intensity change utilizing enhanced satellite derived wind information. M.S. thesis, Dept. of Atmospheric and Oceanic Sciences, University of Wisconsin-Madison, $133 \mathrm{pp}$.

__, and C. S. Velden, 2000: A quantitative look at the relationship between environmental vertical wind shear and tropical cyclone intensity change utilizing enhanced satellite wind information. Preprints, 24th Conf. on Hurricanes and Tropical Meteorology, Fort Lauderdale, FL, Amer. Meteor. Soc., 7A.4, https://ams.confex.com/ams/last2000/ webprogram/Paper12361.html.

— tropical cyclone intensity change utilizing enhanced satellite derived wind information. Preprints, 25th Conf. on Hurricanes and Tropical Meteorology, Boston, MA, Amer. Meteor. Soc., 3C.5, https://ams.confex.com/ams/25HURR/techprogram/ paper_35650.htm.

Gray, W. M., 1968: Global view of the origin of tropical disturbances and storms. Mon. Wea. Rev., 96, 669-700, https://doi.org/ 10.1175/1520-0493(1968)096<0669:GVOTOO>2.0.CO;2.

Griffin, S. M., K. M. Bedka, and C. S. Velden, 2016: A method for calculating the height of overshooting convective cloud tops using satellite-based IR imager and CloudSat cloud profiling radar observations. J. Appl. Meteor. Climatol., 55, 479-491, https://doi.org/10.1175/JAMC-D-15-0170.1.

Guard, C. P., L. E. Carr, F. H. Wells, R. A. Jeffries, N. D. Gural, and D. K. Edson, 1992: Joint Typhoon Warning Center and the challenges of multibasin tropical cyclone forecasting. Wea. Forecasting, 7, 328-352, https://doi.org/10.1175/1520-0434(1992) 007<0328:JTWCAT>2.0.CO;2.

Guimond, S. R., G. M. Heymsfield, P. D. Reasor, and A. C. Didlake, 2016: The rapid intensification of Hurricane Karl (2010): New remote sensing observations of convective bursts from the Global Hawk platform. J. Atmos. Sci., 73, 3617-3639, https:// doi.org/10.1175/JAS-D-16-0026.1.

Harr, P. A., and R. L. Elsberry, 1996: Structure of a mesoscale convective system embedded in Typhoon Robyn during TCM-93. Mon. Wea. Rev., 124, 634-652, https://doi.org/ 10.1175/1520-0493(1996)124<0634:SOAMCS > 2.0.CO;2.

Hodges, K. I., and C. D. Thorncroft, 1997: Distribution and statistics of African mesoscale convective weather systems based on the ISCCP Meteosat imagery. Mon. Wea. Rev., 125, 2821-2837, https://doi.org/ 10.1175/1520-0493(1997)125<2821:DASOAM>2.0.CO;2.

Hoskins, B. J., M. E. McIntyre, and A. W. Robertson, 1985: On the use and significance of isentropic potential vorticity maps. Quart. J. Roy. Meteor. Soc., 111, 877-946, https://doi.org/ 10.1002/qj.49711147002.

Jones, S. C., 1995: The evolution of vortices in vertical shear. I: Initially barotropic vortices. Quart. J. Roy. Meteor. Soc., 121, 821-851, https://doi.org/10.1002/qj.49712152406.

Kaplan, J., M. DeMaria, and J. A. Knaff, 2010: A revised tropical cyclone rapid intensification index for the Atlantic and eastern North Pacific basins. Wea. Forecasting, 25, 220-241, https://doi.org/ 10.1175/2009WAF2222280.1. 
_ , and Coauthors, 2015: Evaluating environmental impacts on tropical cyclone rapid intensification predictability utilizing statistical models. Wea. Forecasting, 30, 1374-1396, https://doi.org/ 10.1175/WAF-D-15-0032.1.

Knabb, R. D., 2008: Hurricane Hernan discussion 12. NOAA/NHC Rep. EP092008, http://www.nhc.noaa.gov/archive/2008/ep09/ ep092008.discus.012.shtml.

_, L. A. Avila, J. L. Beven, J. L. Franklin, R. J. Pasch, and S. R. Stewart, 2008: Eastern North Pacific hurricane season of 2005. Mon. Wea. Rev., 136, 1201-1216, https://doi.org/10.1175/ 2007MWR2076.1.

Knaff, J. A., 2008: Rapid tropical cyclone transitions to major hurricane intensity: Structural evolution of infrared imagery. 28th Conf. on Hurricanes and Tropical Meteorology, Orlando, FL, Amer. Meteor. Soc., 15A.1, http://ams.confex.com/ams/ pdfpapers/137929.pdf.

— S. P. Longmore, and D. A. Molenar, 2014: An objective satellite-based tropical cyclone size climatology. J. Climate, 27, 455-476, https://doi.org/10.1175/JCLI-D-13-00096.1.

Kossin, J. P., 2002: Daily hurricane variability inferred from GOES infrared imagery. Mon. Wea. Rev., 130, 2260-2270, https://doi.org/ 10.1175/1520-0493(2002)130<2260:DHVIFG >2.0.CO;2.

Krishnamurti, T. N., S. Pattnaik, L. Stefanova, T. S. V. Vijaya Kumar, B. P. Mackey, A. J. O'Shay, and R. J. Pasch, 2005: The hurricane intensity issue. Mon. Wea. Rev., 133, 1886-1912, https://doi.org/10.1175/MWR2954.1.

Lander, M. L., 1994: Description of a monsoon gyre and its effects on the tropical cyclones in the western North Pacific during August 1991. Wea. Forecasting, 9, 640-654, https://doi.org/ 10.1175/1520-0434(1994)009<0640:DOAMGA > 2.0.CO;2.

Landsea, C. W., and J. L. Franklin, 2013: Atlantic hurricane database uncertainty and presentation of a new database format. Mon. Wea. Rev., 141, 3576-3592, https://doi.org/10.1175/ MWR-D-12-00254.1.

Merrill, R. T., and C. S. Velden, 1996: A three-dimensional analysis of the outflow layer of Supertyphoon Flo (1990). Mon. Wea. Rev., 124, 47-63, https://doi.org/10.1175/1520-0493(1996) 124<0047:ATDAOT>2.0.CO;2.

Molinari, J., and D. Vollaro, 2010: Rapid intensification of a sheared tropical storm. Mon. Wea. Rev., 138, 3869-3885, https://doi.org/10.1175/2010MWR3378.1.

—, P. Dodge, D. Vollaro, K. L. Corbosiero, and F. Marks Jr., 2006: Mesoscale aspects of the downshear reformation of a tropical cyclone. J. Atmos. Sci., 63, 341-354, https://doi.org/10.1175/JAS3591.1.

_, J. Frank, and D. Vollaro, 2013: Convective bursts, downdraft cooling, and boundary layer recovery in a sheared tropical storm. Mon. Wea. Rev., 141, 1048-1060, https://doi.org/ 10.1175/MWR-D-12-00135.1.

Nguyen, L. T., and J. Molinari, 2015: Simulation of the downshear reformation of a tropical cyclone. J. Atmos. Sci., 72, 45294551, https://doi.org/10.1175/JAS-D-15-0036.1.

_ R. F. Rogers, and P. D. Reasor, 2017: Thermodynamic and kinematic influences on precipitation symmetry in sheared tropical cyclones: Bertha and Cristobal (2014). Mon. Wea. Rev., 145, 4423-4446, https://doi.org/10.1175/MWR-D-17-0073.1.

Nuttall, A. H., 1981: Some windows with very good sidelobe behavior. IEEE Trans. Acoust. Speech Signal Process., 29, 84-91, https://doi.org/10.1109/TASSP.1981.1163506.

Olander, T. L., and C. S. Velden, 2007: The advanced Dvorak technique: Continued development of an objective scheme to estimate tropical cyclone intensity using geostationary infrared satellite imagery. Wea. Forecasting, 22, 287-298, https:// doi.org/10.1175/WAF975.1.
— , and — 2009: Tropical cyclone convection and intensity analysis using differenced infrared and water vapor imagery. Wea. Forecasting, 24, 1558-1572, https://doi.org/10.1175/2009WAF2222284.1. ,$- \ldots$, and J. P. Kossin, 2004: The advanced objective Dvorak technique (AODT): Latest upgrades and future directions. 26th Conf. on Hurricanes and Tropical Meteorology, Miami, FL., Amer. Meteor. Soc., P1.19, https://ams.confex.com/ams/ 26HURR/techprogram/paper_75417.htm.

Pasch, R., 2015: Hurricane Patricia discussion 8. NOAA/NHC Rep. EP202015, http://www.nhc.noaa.gov/archive/2015/ep20/ ep202015.discus.008.shtml.

Rappin, E. D., and D. S. Nolan, 2012: The effect of vertical shear orientation on tropical cyclogenesis. Quart. J. Roy. Meteor. Soc., 138, 1035-1054, https://doi.org/10.1002/qj.977.

Reasor, P. D., M. D. Eastin, and J. F. Gamache, 2009: Rapidly intensifying Hurricane Guillermo (1997). Part I: Lowwavenumber structure and evolution. Mon. Wea. Rev., 137, 603-631, https://doi.org/10.1175/2008MWR2487.1.

_, R. Rogers, and S. Lorsolo, 2013: Environmental flow impacts on tropical cyclone structure diagnosed from airborne Doppler radar composites. Mon. Wea. Rev., 141, 2949-2969, https:// doi.org/10.1175/MWR-D-12-00334.1.

Reynolds, R. W., N. A. Rayner, T. M. Smith, D. C. Stokes, and W. Wang, 2002: An improved in situ and satellite SST analysis for climate. J. Climate, 15, 1609-1625, https://doi.org/10.1175/ 1520-0442(2002)015<1609:AIISAS > 2.0.CO;2.

Riemer, M., M. T. Montgomery, and M. E. Nicholls, 2010: A new paradigm for intensity modification of tropical cyclones: Thermodynamic impact of vertical wind shear on the inflow layer. Atmos. Chem. Phys., 10, 3163-3188, https://doi.org/ 10.5194/acp-10-3163-2010.

Rios-Berrios, R., and R. D. Torn, 2017: Climatological analysis of tropical cyclone intensity changes under moderate vertical wind shear. Mon. Wea. Rev., 145, 1717-1738, https://doi.org/ 10.1175/MWR-D-16-0350.1.

Rogers, R. F., P. D. Reasor, and J. A. Zhang, 2015: Multiscale structure and evolution of Hurricane Earl (2010) during rapid intensification. Mon. Wea. Rev., 143, 536-562, https://doi.org/ 10.1175/MWR-D-14-00175.1.

, J. A. Zhang, J. Zawislak, H. Jiang, G. R. Alvey III, E. J. Zipser, and S. N. Stevenson, 2016: Observations of the structure and evolution of Hurricane Edouard (2014) during intensity change. Part II: Kinematic structure and the distribution of deep convection. Mon. Wea. Rev., 144, 3355-3376, https://doi.org/10.1175/ MWR-D-16-0017.1.

Rotunno, R., and K. A. Emanuel, 1987: An air-sea interaction theory for tropical cyclones. Part II: Evolutionary study using a nonhydrostatic axisymmetric numerical model. J. Atmos. Sci., 44, 542-561, https://doi.org/10.1175/1520-0469(1987) 044<0542:AAITFT $>2.0 . \mathrm{CO} ; 2$.

Rozoff, C. M., C. S. Velden, J. Kaplan, J. P. Kossin, and A. J. Wimmers, 2015: Improvements in the probabilistic prediction of tropical cyclone rapid intensification with passive microwave observations. Wea. Forecasting, 30, 1016-1038, https://doi.org/ 10.1175/WAF-D-14-00109.1.

Ryglicki, D., J. Doyle, Y. Jin, D. Hodyss, and J. Cossuth, 2018: The unexpected rapid intensification of tropical cyclones in moderate vertical wind shear. Part II: Vortex tilt. Mon. Wea. Rev., 146, 3801-3825, https://doi.org/10.1175/MWR-D-18-0021.1.

Sanders, F., and K. A. Emanuel, 1977: The momentum budget and temporal evolution of a mesoscale convective system. J. Atmos. Sci., 34, 322-330, https://doi.org/10.1175/1520-0469(1977) $034<0322:$ TMBATE $>2.0 . \mathrm{CO} ; 2$. 
Shuman, F. G., 1957: Numerical methods in weather prediction: Part II. Smoothing and filtering. Mon. Wea. Rev., 85, 357-361, https:// doi.org/10.1175/1520-0493(1957)085<0357:NMIWPI>2.0.CO;2.

Smith, S. W., 2003: Digital Signal Processing: A Practical Guide for Engineers and Scientists. Elsevier, $650 \mathrm{pp}$.

Stevenson, S. N., K. L. Corbosiero, and J. Molinari, 2014: The convective evolution and rapid intensification of Hurricane Earl (2010). Mon. Wea. Rev., 142, 4364-4380, https://doi.org/ 10.1175/MWR-D-14-00078.1.

Tao, D., and F. Zhang, 2015: Effects of vertical wind shear on the predictability of tropical cyclones: Practical versus intrinsic limit. J. Adv. Model. Earth Syst., 7, 1534-1553, https://doi.org/ 10.1002/2015MS000474.

Torrence, C., and G. P. Compo, 1998: A practical guide to wavelet analysis. Bull. Amer. Meteor. Soc., 79, 61-78, https://doi.org/ 10.1175/1520-0477(1998)079<0061:APGTWA > 2.0.CO;2.

Velden, C. S., and J. Sears, 2014: Computing deep-tropospheric vertical wind shear analyses for tropical cyclone applications: Does the methodology matter? Wea. Forecasting, 29, 11691180, https://doi.org/10.1175/WAF-D-13-00147.1.

_ C. M. Hayden, S. J. Nieman, W. P. Menzel, S. Wanzong, and J. S. Goerss, 1997: Upper-tropospheric winds derived from geostationary satellite water vapor observations. Bull. Amer. Meteor. Soc., 78, 173-196, https://doi.org/10.1175/1520-0477 (1997)078<0173:UTWDFG>2.0.CO;2.

-, T. Olander, and R. M. Zehr, 1998: Development of an objective scheme to estimate tropical cyclone intensity from digital geostationary satellite infrared imagery. Wea.
Forecasting, 13, 172-186, https://doi.org/10.1175/1520-0434 (1998)013<0172:DOAOST>2.0.CO;2.

— — — _ D. Herndon, and J. P. Kossin, 2017: Reprocessing the most intense historical tropical cyclones in the satellite era using the advanced Dvorak technique. Mon. Wea. Rev., 145 , 971-983, https://doi.org/10.1175/MWR-D-16-0312.1.

Wilks, D. S., 2011: Statistical Methods in the Atmospheric Sciences. 3rd ed. International Geophysics Series, Vol. 100, Academic Press, $704 \mathrm{pp}$.

Wirth, V., 2001: Cyclone-anticyclone asymmetry concerning the height of the thermal and the dynamical tropopause. J. Atmos. Sci., 58, 26-37, https://doi.org/10.1175/1520-0469(2001) $058<0026$ :CAACTH $>2.0 . \mathrm{CO} ; 2$.

Wu, C.-C., and K. Emanuel, 1993: Interaction of a baroclinic vortex with background shear: Application to hurricane movement. J. Atmos. Sci., 50, 62-76, https://doi.org/10.1175/1520-0469 (1993) $050<0062:$ IOABVW $>2.0 . \mathrm{CO} ; 2$.

Zawislak, J., H. Jiang, G. R. Alvey III, E. J. Zipser, R. F. Rogers, J. A. Zhang, and S. N. Stevenson, 2016: Observations of the structure and evolution of Hurricane Edouard (2014) during intensity change. Part I: Relationship between the thermodynamic structure and precipitation. Mon. Wea. Rev., 144, 3333-3354, https://doi.org/10.1175/MWR-D-16-0018.1.

Zehr, R., 1989: Improved objective satellite estimates of tropical cyclone intensity. Preprints, 18th Conf. on Hurricanes and Tropical Meteorology, San Diego, CA, Amer. Meteor. Soc., J25-J28. 